\title{
1 Optogenetic perturbations of RNA expression in tissue space
}

2 Ivano Legnini ${ }^{1}$, Lisa Emmenegger ${ }^{1}$, Ricardo Wurmus ${ }^{2}$, Alessandra Zappulo ${ }^{3}$, Anna Oliveras

3 Martinez ${ }^{4}$, Cledi Cerda Jara ${ }^{1}$, Anastasiya Boltengagen ${ }^{1}$, Talé Hessler ${ }^{1}$, Guido Mastrobuoni ${ }^{5}$, 4 Agnieszka Rybak-Wolf ${ }^{6}$, Stefan Kempa ${ }^{5}$, Robert Zinzen ${ }^{3}$, Andrew Woehler ${ }^{4}$ and Nikolaus 5 Rajewsky $^{1}$

7 1. Laboratory for Systems Biology of Gene Regulatory Elements

8 2. Bioinformatics and Omics Data Science

9 3. Systems Biology of Neural Tissue Differentiation

10 4. Systems Biology Imaging

11 5. Proteomic and Metabolomics Platform

6. Organoid platform

Applies to all: Berlin Institute for Medical Systems Biology (BIMSB),

Max Delbrück Center for Molecular Medicine (MDC) in the Helmholtz Association,

*correspondence to rajewsky@mdc-berlin.de

\section{Keywords}

Spatial RNA perturbations, spatial transcriptomics, CRISPR/Cas13, CasRx, optogenetics, single cell, stem cells, organoids, Sonic Hedgehog $(\mathrm{SHH})$ 


\section{Abstract}

2 Quantifying gene expression in space, for example by spatial transcriptomics, is essential 3 for describing the biology of cells and their interactions in complex tissues. Perturbation

4 experiments, at single-cell resolution and conditional on both space and time, are necessary

5 for dissecting the molecular mechanisms of these interactions. To this aim, we combined

6 optogenetics and CRISPR technologies to activate or knock-down RNA of target genes, at 7 single-cell resolution and in programmable spatial patterns. As a proof of principle, we 8 optogenetically induced Sonic Hedgehog $(\mathrm{SHH})$ signaling at a distinct spatial location within 9 human neural organoids. This robustly induced known $\mathrm{SHH}$ spatial domains of gene expression - cell-autonomously and across the entire organoid. In principle, our approach

11 can be used to induce or knock down RNAs from any gene of interest in specific spatial locations or patterns of complex biological systems. 


\section{Introduction}

Within the last decade, novel technologies have enabled sequencing (e.g. Lee et al., 2014, Ståhl et al., 2016, Rodrigues et al., 2019) or imaging (e.g. Chen et al., 2015, Shah et al., 2016) thousands of transcripts in situ, thereby preserving the information of their spatial localization within complex tissues. These approaches may have a profound impact on the way we examine biological systems by allowing, for example, the detection of new types of histopathological signatures in tissue sections (Maniatis et al., 2019), or studying RNA compartmentalization in single cells (Xia et al., 2019). While quantifying and describing spatial RNA expression is helpful for understanding the organization of gene expression in complex tissues (e.g. Nitzan et al., 2019), the ability to perturb RNA expression in tissue space is fundamentally important to dissect the biological function of gene expression in processes and pathways that regulate cell-cell interactions, tissue architecture and homeostasis.

We addressed this problem by establishing an optogenetic system that enables inducible, targeted and localized RNA perturbation in a variety of biological systems. Several efforts have been made in this direction by combining light-responsive protein modules (Kennedy et al., 2010, Renicke et al., 2013, Kawano et al., 2015) with CRISPR/Cas9 (Nihongaki et al., 2015a, Zhou et al., 2017), to achieve irreversible genetic mutations. However, the readouts are difficult to interpret within a simple functional study due to mutational heterogeneity and limited efficacy. Another approach, light-inducible knock-downs, has only been achieved in cell lines, for example with photo-caged oligonucleotides/siRNAs (e.g. Mikat et al., 2007), or more recently with a genetically encoded optogenetic RNA interference (RNAi) system (Pilsl et al., 2020). While these methods are efficient and allow for precise temporal control in cell lines, their efficacy is unknown in more complex tissues and may be limited. For example, the RNAi approach used in Pilsl et al., is constitutively active in the dark and is inactivated by photo-stimulation, making it challenging to spatially control the knock-down of a given target in an organoid or in vivo. Furthermore, RNAi in general is known to suffer from offtarget effects.

On the other hand, gene activation based on light-inducible transcription (Polstein et al., 2015, Nihongaki et al., 2015b, Nihongaki et al., 2017, De Santis et al., 2021) has been effectively used in cell culture and sometimes in organotypic culture and even in vivo (Yamada et al., 2018). 
1 Therefore, to develop a flexible system that allows for both light-inducible transcription and knock-down of target genes with high resolution in space and time, we combined optogenetic transcription (Nihongaki et al., 2017, Yamada et al., 2018, De Santis et al., 2021) with the recently discovered CRISPR/Cas13 system (Abudayyeh et al., 2017, Cox et al., 2017, Konerman et al., 2018), which can be programmed to target and destroy RNA with high specificity. This combination was implemented by designing synthetic promoters to maximize transcriptional activity while minimizing leakage without light activation. We demonstrate that our combined approach enables effective (and reversible) overexpression (for example, up to 800-fold for messages with low endogenous expression) and 50-70\% knock-down of reporter and endogenous transcripts in cultured cells and organoids, at single-cell resolution. We performed genome-wide mass-spec experiments which demonstrated the high specificity of these knock-downs.

To show that our approach can indeed perturb biological function of gene expression, we chose to target Sonic Hedgehog $(\mathrm{SHH})$ signaling. $\mathrm{SHH}$ is a well-studied morphogen that is central to a variety of biological processes, including dorsoventral patterning of the developing neural tube in vertebrates (Ribes and Briscoe, 2009). SHH synthesis was effectively "printed" onto a cellular layer by light induction, stimulating localized expression of target genes as observed by immunostainings and spatial transcriptomics. We developed neural organoids to mimic aspects of the neural tube and we optogenetically induced $\mathrm{SHH}$ in a pole of these organoids, which was sufficient to robustly establish spatial domains of gene expression - cell-autonomously and across the entire organoid. This setup might prove immensely useful for studying the properties of $\mathrm{SHH}$ signaling and other signaling molecules in vitro, as well as to engineer 3D cellular models with complex spatial patterning modalities.

Having shown that our approach is specific, can be induced at single-cell resolution, and perturbs biological function of gene expression, we sought to design a system to deliver light activation to single cells simultaneously, at programmable, multiple locations within a complex tissue or organoid. Therefore, we constructed a Digital Micromirror Device (DMD) microscope combined with a cell culture chamber for live-cell stimulation. Programming is made easy through a micromanager/ImageJ-based graphical user interface (GUI). We show that this system allows to "print" complex patterns of gene expression. 
1 In principle, our approach can be used to induce or knock down RNAs from any gene of interest in specific spatial locations or patterns of complex biological systems. We also present a careful discussion of its potential and limitations.

\section{Results}

In order to perturb RNA expression with spatial resolution, we adopted, optimized and constructed a variety of tools based on the combination of optogenetic modules, to allow spatial control with photo-stimulation (pMag/nMag and CRY/CIB), and gene perturbation modules, to allow gene activations (CRISPR/Cas9, TetON and Cre/Lox systems) and knock-downs (CRISPR/Cas13). Initial attempts at rendering Cas13 proteins light-responsive were not successful (however, we report several functional split sites for Cas13b, as well as functional Cas13b fusions with RNA silencing domains from GW182 proteins and other constructs with distinct and intriguing properties in a supplementary note and in Figures S12). Instead, a different strategy where we designed synthetic promoters to couple lightinducible gene transcription to CasRx transcription was successful.

Light-inducible gene activation combined with CasRx transcription for optogenetic RNA targeting.

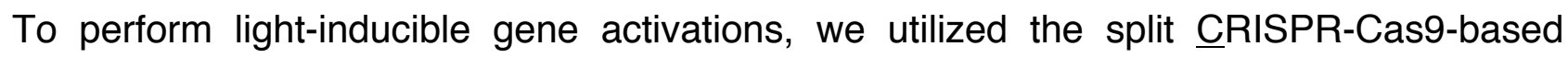
Photoactivatable Iranscription System (split CPTS2.0, hereafter referred to as SCPTS, Nihongaki et al., 2017), which consists of an enzymatically dead Cas9 (dCas9) that has been split into $\mathrm{N}$ - and $\mathrm{C}$-terminal domains, and fused to the photoinducible dimerization moieties pMag and nMag. Blue light triggers pMag-nMag dimerization, thus reconstituting dCas9, which then binds at the targeted promoter according to the loaded guide RNA (sgRNA). This SCPTS system activates transcription in the vicinity (CRISPRa) through a VP64 activation domain fused to the C-terminal Cas9 fragment and p65 and HSF1 domains fused to an MCP moiety that associates with the MS2-stem-loops engineered into the sgRNA (Fig. 1a). This system has been shown to be a potent transcriptional activator under blue light illumination (Nihongaki et al., 2017).

We combined an SCPTS module with a CRISPR/CasRx module (Fig. 1a-b), such that photo-stimulation controls CasRx synthesis to induce targeted RNA knock-downs. For 
1 programming the SCPTS system to stimulate CasRx synthesis upon photo-stimulation, we first optimized the system in the context of HEK cells transfection. To do so, we designed a custom programmable LED board (Methods), which accommodates a 96-well cell culture plates and can be used in a cell culture incubator. Levels of induction of transcription were comaprabel to Nihongaki et al. (2017, Fig. S2a). We then tested a promoter/sgRNA pair, previously used in a similar context (Gal4/UAS, Nihongaki et al, 2015b), and additionally designed two synthetic promoters (CRISPRa Synthetic Promoter - CaSP1 and 2, partially based on Loew et al., 2010, Fig. 1b) to drive CasRx transcription. We transfected HEK cells with the plasmids encoding the transcription system, the sgRNA and a CasRx-T2A-GFP cassette under the control of one of the three promoters, and imaged GFP over time upon photo-stimulation (Fig. 1c, S2b). Both synthetic promoters CaSP1 and CaSP2 are more active than the UAS promoter (Fig. 1d). CaSP1 induced a 45-fold-change activation after $50 \mathrm{~h}$ illumination over the non-targeting guide control, with $\sim 16 \%$ leakage in the dark. In contrast, CaSP2 elicited a 21-fold induction, with a leakage of $\sim 9 \%$ in the dark. To control for unspecific effects of light stimulation, we used a constitutive CasRx-T2A-GFP cassette under a strong EF1a promoter, observing an increase of GFP over 24h of illumination, with no substantial difference between light and dark conditions (Fig. S2c). Representative images of the SCPTS/Cas13 photo-stimulation are shown in Fig. 1e. Two more systems, based on a light-inducible TetON transcription system (PA-TetON, Yamada et al., 2018) and a light-inducible Cre/Lox recombination system (PA-Cre/Lox, De Santis et al., 2021) are described in a supplementary note and in Figure S2d-e. Finally, we confirmed the microscopy-based GFP quantifications for all CasRx expression systems with flow cytometry (Fig. S2f-i).

Light-inducible knock-down of reporter and endogenous transcripts.

We next tested the efficacy of constitutively expressed or light-inducible CasRx in knocking down reporter and endogenous transcripts (Fig. 2a). We constructed a Tet-ON RFP reporter and assessed its knock-down with a constitutively expressed CasRx. CasRx was able to efficiently target RFP with a single guide RNA (PS18 adopted from Abudayyeh et al., 2016 for practical reasons; hereafter referred to as "RFP guide RNA"; Fig. 2b). However, GFP (which tags the CasRx cassette) was also strongly depleted for reasons that we do not fully understand (Fig. S3a). We investigated this effect further and report the results in the 
supplementary note and Fig. S3b-c. To rule out the occurrence of global off-targeting effects, we performed genome-wide mass-spectrometry-based quantitative proteomics. The only proteins with a statistically significant fold change larger than 2 when comparing cells transfected with a non-targeting guide (NT) vs. an RFP-targeting guide were RFP, CasRx and GFP (Fig. 2C). We also sequenced total RNA and found only six differentially expressed transcripts: four were highly homologous to $18 \mathrm{~S}$ ribosomal RNA, which are typical artifacts of the ribodepletion, and the remaining two were RFP and CasRx-GFP, with only RFP having a fold-change larger than 2 (Fig. S3d). In summary, genome-wide protein and RNA quantification both demonstrated high specificity of our knock-down approach.

Given the targeting efficacy and specificity of the constitutive CasRx, we tested whether a light-induced CasRx is also capable of specifically knocking down RFP. We transfected HEK cells with the SCPTS CasRx systems together with either a non-targeting or an RFPtargeting guide and observed RFP knock-down efficiencies of $40-60 \%$ in the lit state, with residual activity of $10-30 \%$ in the dark state, depending on the construct (Fig. 2d). As before, GFP was unexpectedly depleted when CasRx was programmed to target RFP (Fig. S3e). We then tested the CaSP2-CasRx system, which provides a good trade-off between targeting efficacy and leakage, on endogenous targets. We designed two guide RNAs complementary to the circular RNA CDR1as and adopted a previously published sequence for the STAT3 mRNA (Konerman et al., 2018) and validated them with a constitutive CasRx (Fig. S3f). We transfected one CDR1as guide and the STAT3 guide together with the SCPTS-CaSP2-CasRx system, stimulated the cells with blue light for 24-36 hours and performed qRT-PCR for target quantification. As shown in Fig. 2e, we achieved $\sim 71 \%$ and $\sim 45 \%$ knock-down for CDR1as and STAT3 respectively, with 24\% and $11 \%$ leakage.

\section{Spatial gene perturbations.}

In order to leverage the potential of optogenetic RNA perturbations, we not only need programmable activations and knock-downs, but also the means to program spatial stimulations. To this end, we tested three different approaches (Fig. 3a).

First, we applied a laser-printed photomask between the light source and cells that express light-inducible CasRx cassettes (Fig. 3b and S4a). This setup is simple to set up, as a laser printer is sufficient to engrave the desired pattern on a plastic sheet, which is then attached below a cell culture plate. The plate is then placed on top of a LED array and light stimulation 
is provided for the desired time (e.g. 6-50h, as in Fig. S1, 1 and 2). The array is capable of generating complex patterns of activation with a resolution on the order of hundreds of micrometers, provided by the photomask (Fig. 3b). We noticed that the induction diffuses over the edges of the photomask, possibly due to reflection and refraction within the wells. Next, we tested if precise spatial activation of CasRx can be achieved at single-cell resolution. To this aim, we employed a laser scanning-based photo-stimulation approach, and we performed live-cell imaging with a laser scanning confocal microscope. We induced CasRx expression from the light inducible Cre/Lox system by scanning a region of interest (ROI) containing a single-cell. Using this approach, we successfully stimulated a single cell in a field of view (FOV) containing several cells. (Fig. 3c).

Lastly, we constructed a Digital Micromirror Device (DMD) microscope, combined with a cell culture chamber for live-cell stimulation and imaging, that allows to program spatial activation patterns by a simple micromanager/lmageJ-based graphical user interface (GUI). We describe the details for building and programming this "point-and-shoot" setup in the methods section. Within the GUI, we can draw ROls in any shape and number, which will then be illuminated by turning on the associated micromirrors. With this setup, we can program complex patterns of spatial stimulation, for example multiple and different ROls at once (Fig. 3d).

Optogenetic stimulation of the SHH pathway in hiPSCs.

To test the ability of our setup to perturb biologically relevant processes, we focused on the induction of Sonic Hedgehog in hiPSCs (Fig. 4a). We first designed three sgRNAs for activating the SHH promoter with the SCPTS system (Fig. S5a). We transfected HEK293T cells with all the SCPTS modules and each sgRNA, then quantified SHH mRNA expression after 24 hours of photo-stimulation. Guide 1 was the most efficient, increasing SHH mRNA expression ca. 800-fold over a non-targeting guide (Fig. S5b). In addition, we designed guides for another morphogen involved in neurodevelopment, BMP4 (Fig. S5c). Guide 3 was the most effective, inducing a 4-fold increase in BMP4 mRNA levels (Fig. S5d). Leakage was approximately $5 \%$ for $S H H$ guide 1 and $30 \%$ for BMP4 guide 3 . We note that BMP4 was already expressed in HEK cells, which is likely a reason for the lower induction and higher leakage. We assessed whether the induced $\mathrm{SHH}$ exerted its biological activity by stimulating the expression of its targets, upon transfection of the SCPTS system in hiPSCs. 
1 We measured FOXA2, FOXG1, NKX2-1, NKX6-2 and OLIG2 expression after 24, 48 and 72 hours of stimulation. $\mathrm{SHH}$ reached its highest level of activation at 24 hours and decreased at 48 and 72 hours (Fig. S5e-f). FOXA2, NKX6-2 and OLIG2 were significantly upregulated upon light stimulation in neural induction media, but not in stem cell media (Fig. S5e-f). Light-inducible activation of the $\mathrm{SHH}$ promoter is therefore sufficient to stimulate $\mathrm{SHH}$ transcription and exert a detectable biological effect by inducing the expression of some of its known targets. In parallel, we used the PA-Cre/Lox system to generate a stable hiPSC line that can overexpress a NeonGreen-SHH cassette upon light stimulation and doxycycline treatment (SHH-GFP for simplicity, De Santis et al., bioRxiv 2021, suppl. video 1). With this system, we observed stronger $S H H$ mRNA induction upon light stimulation, but also higher leakage; the same was true for its targets (Fig. S4e-g). Robust expression of FOXA2 was visible at the protein level after inducing $\mathrm{SHH}$ for 6-7 days in restricted groups of cells with the DMD setup (Fig. 4b).

To systematically profile spatial gene expression upon spatial gene perturbations, we established a method to transfer cultured cells on a spatial transcriptomic slide (10X Visium). We adapted hiPSC culture and photo-stimulation to cell culture inserts, which consist of a PET membrane held by a plastic scaffold within a cell culture dish. The membrane can be cut from the scaffold, transferred onto a slide and removed after cell fixation (Fig. 4a,c and $\mathrm{S} 5 \mathrm{~h})$. We used this system to probe the gene expression response to the induction of $\mathrm{SHH}$ in the center of the membrane for a time course of 120 hours, with the PA-Cre/Lox system. Since the RNA capture was not homogeneous, yielding vastly different UMI counts across the capture area (Fig. S4i), we merged the transcript counts for a set of concentric circles, with the inner circle enclosing the induced area and the outer ones placed at increasing distances (Fig. 4d). We examined a gene set comprising $\mathrm{SHH}$ and its targets, and retrieved a peak of expression in the inner part of the membrane for all time points after induction, as compared to randomized controls (Fig. $4 \mathrm{e}$ and S5j-I). We note that the raw transcript counts for these transcripts was low (globally, in the range of tens or hundreds), and dominated by $\mathrm{SHH}$ at the shortest time-point. Looking at other genes involved in the SHH pathway, we found that the receptor PTCH1 was strongly upregulated in the proximity of the $\mathrm{SHH}$ signal at $120 \mathrm{~h}$, as, to a lesser extent, its interaction partner SMO (Fig. S5I). 
SHH induction produced a biological response in hiPSCs only detectable with sensitive techniques in short time frames (by qRT-PCR and to a lesser extent by spatial transcriptomics), and becoming more robust over time with the FOXA2 protein becoming detectable 6-7 days after the stimulation. To overcome the constraint of a 2D system which has limited endurance in culture and poor physiological resemblance to a developing tissue, we devised a protocol for producing 3D neural organoids, partially based on previous attempts at mimicking the dorsal-ventral patterning of the caudal part of the neural tube in vitro (Zheng et al., 2019). We used laser scanning to induce SHH expression in a pole of embryoid bodies grown for 4 days (Fig. $4 f$ and suppl. video 2 ), then supplemented the medium with retinoic acid for 5 days and allowed them to grow and differentiate for an additional 7 days (Fig. S5n). We tracked NeonGreen fluorescence to assess spread and location of SHH expressing-cells in whole-mount fixed organoids, and noticed that some of them spread out from the induced pole, likely due to cell divisions and migration. Nevertheless, overall the organoids retained a polarized $\mathrm{SHH}$ pattern, which induced a robust and spatially restricted expression of FOXA2, whereas non-induced organoids produced neither detectable NeonGreen nor FOXA2 (Fig. S40). We stained adjacent organoid slices for $\mathrm{SHH}$ targets known to be induced in different neural tube domains at increasing distance from the $\mathrm{SHH}$ source (Ribes and Briscoe, 2009) and observed that FOXA2 and OLIG2 established, as they should, mutually exclusive expression domains, with FOXA2 being activated in the proximity of SHH-producing cells and OLIG2 further away (Fig. 4g). NKX6-1 expression instead encompassed both FOXA2 and OLIG2 domains, but was restricted to cells located at the exterior of neuroepithelial loops (Fig. $4 \mathrm{~g}$ ). We conclude that optogenetic patterning of neural organoids through localized $\mathrm{SHH}$ induction allows to establish spatially restricted patterns of RNA expression, marked by transcription factors known to specify distinct populations of progenitor cells in vivo.

\section{Discussion}

The pursuit of imaging RNA/protein expression in tissues dates back to decades ago. In situ hybridization and immunostainings have been instrumental to understand the complex interplay of cells in tissues in health, disease and development. In the past few years, spatial investigation of RNA expression has made huge advances, with new technologies becoming available for high-throughput spatial transcriptomic profiling (see Moses and Pachter, 2021 
for a tour of the museum of spatial transcriptomics). We believe that one of the next frontiers is to marry high-throughput spatial transcriptomics with precise and spatially resolved functional perturbations. To this aim, we developed an experimental setup which allows the following: 1) choose a gene or a gene set of interest, 2) perturb it (by RNA knock-down, activation, or both) in a specific area of interest of a 2D or 3D cellular model and 3) measure the impact of the perturbation in the transcriptomic and physical space. With this work, we both provide a general blueprint as well as tools for performing these experiments. All tools are made freely available.

We first implemented and optimized available tools for optogenetic gene activation, and at the same time devised molecular tools to perform RNA knock-downs at single-cell resolution. We therefore combined optogenetics, to provide spatial control, with CRISPR/Cas13, to perform programmable knock-downs. To do so, we used a CRISPRabased transcription system (Nihongaki et al., 2017), in addition to a Tet-ON transcription system (Yamada et al., 2018), and a Cre/Lox system (De Santis et al., bioRxiv 2021), which are more extensively described in the supplement. We combined these with different CasRx expression cassettes and found that they could induce CasRx synthesis upon photostimulation, with a tradeoff between expression level and background. For example, the CRISPRa system combined with our CaSP1 promoter was very effective upon light stimulation, but it also had high background. CaSP2 had lower efficiency, but also lower leakage. This is common for inducible systems, and one has to adjust the experimental conditions to reach a predetermined goal. In this case, one can titrate the minimum amount of CasRx required for efficient knock-down of a given target, and then adjust the experimental setup to have the lit state exceeding that threshold. These systems enabled silencing of reporter and endogenous targets with varying efficiencies and leakage. Further improvements may be possible, for example by using guide RNA arrays instead of single guides (Konermann et al., 2018).

To imprint gene expression patterns on a cellular "canvas", we tested different means for spatial stimulations, including photomasks, laser scanning and digital light projection. While fast and comparatively easy, using a photomask in between a light source (e.g. a LED array) and the specimen lacks in resolution and accuracy. To overcome these limitations, we used a laser scanning setup that provides enough resolution, precision and minimum background noise to stimulate a single cell. A key limitation of the scanning-based system is that only a 
1 single volume of a predefined shape can be stimulated at a time (while, with more sophisticated setups, multiple or complex patterns could be stimulated sequentially). A promising and extremely versatile alternative is to employ a DMD setup, which uses a programmable array of micro-mirrors (as for example in Yamada et al., 2018), to simultaneously illuminate multiple and complex ROls. We constructed such a setup with a commercial digital light processing projector and showed that we can project complex stimulation patterns on cells, inducing CasRx expression in defined ROls which can be drawn in a convenient GUI.

Spatial perturbations of RNA may be extremely useful in studying numerous biological processes. For example, overexpression of oncogenes or knock-down of tumor suppressors in a single cell or in defined cell types within organoids may be used as a novel model for tumorigenesis, by examining proliferation and migration of the perturbed cells in the tissue with extreme spatiotemporal control. Another interesting application is the study of developmental processes involving cell-cell interactions. For example, inducing or knocking down signaling molecules or receptors can help understanding the principles and kinetics of cellular interactions in a simplified setup, where the position of sender or receiver cells can be programmed by photo-stimulation (e.g. Rogers et al., 2020). As a paradigm of this sort of application, we focused on the Sonic Hedgehog pathway. SHH is a well-known morphogen produced during vertebrate development by the notochord and the floor plate of the neural tube. It is released in the extracellular space, where it forms a gradient along the ventral-dorsal axis, which specifies the fate of progenitor cells located at increasing distances from the source of the signal. To imitate this process in vitro, we locally induced $\mathrm{SHH}$ in an "artificial organizer" and measured its effect on gene expression over time. There have been several studies on $\mathrm{SHH}$ signaling in vitro, by means of treatment with a recombinant protein (Kutejova et al., 2016), its overexpression (Li et al., 2018) or fusing a SHH-expressing spheroid with organoids (Cederquist et al., 2019). Very recently, De Santis et al. (bioRxiv, 2021) used a light-inducible Cre/Lox system to overexpress SHH in human induced pluripotent stem cells and showed that this approach can induce a strong cellautonomous and non-autonomous response. We adopted the same system, as well as a CRISPRa-based system to activate the endogenous SHH locus, and combined it with spatial transcriptomics, by establishing a protocol for transferring a cell monolayer to a glass slide suitable for analysis by Visium (10X). We could observe an induction of the pathway in this setup, but with limited resolution and sensitivity. We therefore established a protocol for 
1 producing human neural organoids, which we expected to reflect more closely the

2 physiological properties of a developing tissue. We optogenetically induced $\mathrm{SHH}$ in a

3 restricted group of cells within these organoids. This was sufficient to robustly induce classic

4 patterns of $\mathrm{SHH}$ signaling across the entire organoids. We believe that this setup might

5 prove extremely useful for studying spatio-temporal properties of $\mathrm{SHH}$ signaling or other

6 signaling pathways, as well as for generating new organoid models with complex and

7 controlled spatial patterns of gene expression.

8 In summary, implementing our approach to perturb RNA expression in tissue space is 9 practicable within a few weeks in cell lines and organoids in a simplified and general setup, 10 which includes: a) designing and cloning guide RNAs or expression cassettes within the 11 available plasmids (Table S2, soon in Addgene), b) transfecting cells for transient 12 perturbations or generating stable lines for long-term experiments / organoid generation, c) 13 activating the perturbations with one of the proposed photostimulation setups, for which 14 detailed construction, programming and usage procedures are provided in the Methods. 


\section{Acknowledgements}

2 We thank Gwendolin Matz and Ruth Alcaraz Pareja for help with cloning and cell culture, Margareta Herzog and Alex Tschernycheff for administration, Salah Ayoub for RNAsequencing experiments, Sebastian Ehrig for helping with photomasks printing and for casting the PDMS supports for organoids induction, Jonathan Froehlich, Giuseppe Macino, Andreas Möglich and Peter Hegemann for useful discussions, Anna Löwa (BIMSB Organoid platform), Sebastian Diecke and Ralph Kuhn (fMDC stem cell core facility) for advice on hiPSC culture and transfections, Omar Abudayyeh, Jonathan Gootenberg and Feng Zhang for sharing plasmids and advice regarding Cas13b, Riccardo De Santis for sharing the Cre/Lox plasmids and advice regarding the Cre experiments, Yuta Nihongaki for advice regarding CPTS/split-CPTS optimization, Itaru Imayoshi and Mayumi Yamada for sharing the PA-TetON plasmids, Heiko Lickert for sharing the XM001 hiPSC line, the whole N. Rajewsky lab for discussions, support and constructive feedback, particularly David Koppstein for reading the manuscript, Tatiana Borodina, Caroline Braeuning, Thomas Conrad, Daniele Yumi Sunaga-Franze, Kerim Secener, Jeannine Wilde (BIMSB sequencing facility) for sequencing, Visium and FACS experiments. All illustrations were created with BioRender.com.

\section{Funding}

IL was a recipient of an EMBO Long Term Fellowship when this work was started (ALTF 1235-2016). Third party funding came from the Deutsche Forschungsgemeinschaft (DFG \#RA838/5-1), Berlin Institute of Health (BIH \#CRG2aTP7), Deutsches Zentrum für HerzKreislauf-Forschung (DZHK \#81X2100155).

\section{Author contributions}

NR and IL conceived the original idea. NR supervised the whole project, giving input about experiments and analyses. IL wrote the first manuscript draft with input and feedback from all authors, NR revised and finalized the manuscript. IL designed and supervised all experiments with input from NR, performed all Cas13 experiments in HEK cells, cloned most of the plasmids, performed RNA extractions and qPCRs, performed most of the inductions with the LED board, the confocal and the DMD setups, analyzed qPCR, imaging, RNA-seq 
and Visium data. LE performed all experiments in hiPSCs, RNA extractions, qPCRs, immunostainings and optimized the Visium protocol from 2D culture. $A B$ optimized the Visium protocol for 2D culture with LE and produced all Visium libraries. CCJ optimized and performed live cell imaging of GFP and RFP in HEK cells. TH generated the PA-Cre/Lox HEK cell lines, cloned some of the plasmids, performed RNA extractions and qPCRs and assisted LE with immunostainings. GM performed the proteomics experiment and analyses, under the supervision of SK. AOM optimized and performed CasRx inductions with the confocal microscope setup with IL. ARW supervised the work done with hiPSCs and performed cell and organoid culture. AW constructed the DMD setup, wrote the code for controlling spatial stimulations and optimized CasRx and $\mathrm{SHH}$ inductions with this setup. $\mathrm{RW}$ contributed at the initial stage of the project designing, producing and programming the LED board for whole-well stimulations and assisting IL with the first induction experiments. AZ developed the protocol for generating neural organoids, performed $\mathrm{SHH}$ inductions with $\mathrm{IL}$, immunostainings and imaging under the supervision of $\mathrm{RZ}$.

\section{Methods}

Cell culture, transfections and cell lines generation.

HEK293T cells were cultured in DMEM (high-glucose, with glutamax and pyruvate, Thermo Fischer \#11360872) supplemented with 10\% Tet-free FBS (PAN biotech., \#P30-3602) in absence of antibiotics at $37^{\circ} \mathrm{C}$ with $5 \% \mathrm{CO}_{2}$. They were split every $2 / 3$ days with $0.05 \%$ trypsin in $10 \mathrm{~cm}$ cell culture dishes. For transfection experiments shown in figure 1, 30,000 cells were seeded the evening before transfection in $70 \mu \mathrm{l}$ medium on white 96-well, clearbottom plates (Corning \#3610). The morning after, a mix comprised of $12 \mu$ Optimem (Thermo Fisher, \#31985062), 25 ng Luciferase-encoding plasmid, 150 ng guide RNAencoding plasmid and 150 ng Cas13-encoding plasmid or 2 times 100 ng of each SplitCas13-encoding plasmid was mixed with $25 \mu$ Optimem and $0.5 \mu$ l Lipofectamine 2000 (Thermo Fisher \#11668019), incubated at room temperature for 10 minuets and then pipetted onto the cells. Light stimulation was started 6 hours post-transfection and luciferase assay was performed in the same plate 24 hours post-induction by removing $50 \mu \mathrm{l}$ medium, adding $75 \mu$ l Luciferase assay buffer (Promega Dual-Luciferase Reporter Assay System, \#E1910), incubating 10 minutes at room temperature, then reading Firefly luciferase, then $75 \mu$ Stop\&Glo buffer, incubating 10 minutes at room temperature, then reading Renilla 
luciferase. Plate readings were performed in a Tecan M200 infinite Pro plate reader with two-seconds integration for luciferase measurement.

For transfection experiments shown in figures S1, 1, 2 and 3, 25,000 cells were seeded the evening before transfection in $70 \mu \mathrm{l}$ medium on black 96-well, clear-bottom plates (Corning \#3904). The morning after, a transfection mix comprised of $25 \mu$ l Optimem, $0.4 \mu \mathrm{l}$ P3000 and 100-300 ng plasmid DNA was pooled with $25 \mu$ Optimem and $0.3 \mu$ L Lipofectamine 3000 (Thermo Fisher \#L3000001), incubated for 10 minutes at room temperature and then pipetted onto the cells. Light stimulation was started 6 hours post-transfection and live-cell imaging was performed at 24 hours post-induction unless differently indicated (e.g. for the time-course in figure 1).

Transfections for the RNA-seq and proteomics experiments shown in figure 2 were performed in 6-well plates with 1 million HEK293T cells per well. The morning after seeding, a mix comprised of $250 \mu \mathrm{l}$ Optimem, $800 \mathrm{ng}$ ePB Puro TT RFP plasmid, $1000 \mathrm{ng}$ guide RNAencoding plasmid and 1000 ng Cas13-encoding plasmid, $8 \mu$ p3000 reagent and $2 \mu l$ doxycycline $(1 \mathrm{mg} / \mathrm{ml})$ was mixed with $250 \mu \mathrm{l}$ Optimem and $6 \mu$ Lipofectamine 3000, incubated at room temperature for 10 minutes and then pipetted onto the cells. Cells were harvested 36h post-transfection for RNA extraction with home-made trizol or for protein purification, as described later in the RNA-seq and proteomics sections.

Transfections for generating the Cre/Lox CasRx line were performed in 12-well plates seeded with 100,000 HEK293T cells and the day after transfected with $250 \mathrm{ng}$ ePB-PA-Cre plasmid, $500 \mathrm{ng}$ LoxP-CasRx plasmid, $125 \mathrm{ng}$ hyperactive transposase plasmid, $100 \mu \mathrm{l}$ Optimem and $2.5 \mu \mathrm{l}$ p3000 reagent, mixed with $100 \mu \mathrm{l}$ Optimem and $1.5 \mu \mathrm{l}$ Lipofectamine 3000 reagent, incubated for 10 min at RT and then pipetted onto the cells. After 3 days posttranfection, cells were split into two wells of a new 6-well plate and selected with puromycin $(1 \mu \mathrm{g} / \mathrm{ml})$ and blasticidin $(5 \mu \mathrm{g} / \mathrm{ml})$ for a week. RFP ${ }^{+} / \mathrm{GFP}^{-}$cells were further FACS-sorted for increasing the purity of the population.

HiPSCs (XM001 line, Wang et al., 2018) were cultured in E8 flex media with supplement (Thermo Fisher \#A2858501) at $37^{\circ} \mathrm{C}$ in hypoxia (5\% O2) conditions, passaged every 3-4 days with accutase, and seeded on Geltrex- (Gibco \#A1413302) coated plates. To promote their attachment to the plates, cells were kept in E8 flex media supplemented with $10 \mu \mathrm{M}$ Rho-associated protein kinase (ROCK) inhibitor. Media was changed to E8 flex without ROCK inhibitor within the next $24 \mathrm{~h}$ from plating. 
1 The PA-TetON CasRx cell line was produced with two lentiviruses produced in HEK293T cells with the PA-TetON plasmid (Yamada et al., 2018) and the TRE-CasRx plasmid, two packaging plasmids (Addgene \#8454 and 8455). 50,000 HEK293T were transduced with a $\mathrm{MOI}$ of 10 of each lentivirus in a 24-well format and then selected for blasticidin expression $(5 \mu \mathrm{g} / \mathrm{ml})$ for one week.

HiPSCs transfections were conducted using Lipofectamine Stem (Thermo Fisher \#STEM00001). For transfections experiments regarding the timecourse of $\mathrm{SHH}$ activation shown in Figures 4 and S5, hiPSCs colonies at $70-80 \%$ confluency were dissociated to single cells with accutase. 250,000 cells were then resuspended in 100ul of E8 flex with $10 \mu \mathrm{M}$ ROCK inhibitor and seeded on black 96-well plates, previously coated with Geltrex. After 2-3 h, when cells got attached to the wells, media was changed with $50 \mu$ of E8 flex with ROCK inhibitor. The transfection was performed as follows: for one sample, $1.2 \mu \mathrm{l}$ of Lipofectamine were mixed with $25 \mu \mathrm{l}$ of Optimem and $500 \mathrm{ng}$ of total plasmids were diluted in $25 \mu \mathrm{l}$ of Optimem. Diluted Lipofectamine and diluted plasmids were then combined at a ratio $1: 1$, incubated at room temperature for 10 minutes and $50 \mu \mathrm{l}$ pipetted drop by drop on top of the cells. Transfection efficiency was assessed by including a control plasmid encoding for constitutive GFP (pmax-GFP, Lonza, \#V4YP-1A24). Media was changed within 7-8 hours after transfection to E8 flex and, prior to light induction, gradually replaced by neural induction media "COM1", whose composition is the following: DMEM-F12 (Thermo Fisher \#11320033), N2 supplement (Thermo Fisher \#17502048), Neurobasal (Thermo Fisher \#21103049), B27-vitamin A (Thermo Fisher \#12587010), 1X Penicillin/Streptomycin, Glutamax (Thermo Fisher \#35050061), 2-mercaptoethanol, vitamin C, CDLC (chemically defined lipid concentrate) and insulin. The reason of this media switch was due to the fact that E8 flex contains basic fibroblast growth factor (FGF2), which is a strong inhibitor of the SHH signalling pathway (Fogarty et al., 2007). Light stimulation was started within 15 hours post-transfection, using blue LED array. Cells were harvested after each time point of light stimulation and lysed with $100 \mu$ l home-made Trizol. RNA was then extracted with the Zymo RNA extraction kit (Zymo \#R2051). cDNA preparation and qRT PCR were performed as described in paragraph "RNA extraction, qRT-PCRs".

The plasmids encoding the SCPTS 2.0 systems were generated as previously described with standard molecular cloning approach (Nihongaki et al., 2017), and with the aim to produce stable iPSC lines, the modules in these plasmids (split Cas9, MCP, sgRNA 
cassette) were PCR-amplified and recloned into 2 lentiviral plasmids (pLKO.1 neo, Addgene \#13425; pLJM-EGFP, Addgene \#19319) and, from the latter, they were subcloned into two PiggyBac transposons vectors. With the SCPTS2.0 system we did not succeed in generating stable lines, neither by lentiviral infections nor by transposase/PiggyBac strategy: in both cases, we experienced a full cell mortality, perhaps due to some forms of toxicity of the system. On the other hand, we managed to generate stable iPSCs Cre/Lox SHH and Cre/Lox CasRx lines by transfecting hiPSCS with PiggyBac vectors and the transposase enzyme. In this regard, 400,000 hiPSCs were plated in a Geltrex-coated well of a 12 wellformat plate and transfected with lipofectamine. Herein, 400ng of each transposon- one carrying the "TRE- CRE split1 nMag -T2A/P2A- pMag CRE split2" and the other carrying the "CAG-loxP-RFP-loxP-CasRx or SHH cassettes"- were combined with 200 ng of hyperactive transposase and diluted in $72 \mu \mathrm{l}$ of Optimem. Diluted DNA was then mixed with lipofectamine $(3 \mu \mathrm{l})$, also diluted with Optimem $(72 \mu \mathrm{l})$ and incubated at RT for 10 minutes. More specific details of the transfection protocol are outlined above. Media was changed to E8 flex after $5 \mathrm{~h}$ from transfection. Cells were let recover for 4 days and antibiotics selection was then started, immediately after splitting hiPSCS: $1 \mu \mathrm{g} / \mathrm{ml}$ of puromycin and $2 \mu \mathrm{g} / \mathrm{ml}$ of blasticidin were added to E8 flex medium. Cells were kept under antibiotics selection for 10 days. As a readout of successful integration of the transposons cassettes, RFP signal was checked.

Organoids differentiation.

HiPSCs were cultured in E8 flex medium (Gibco \#A14133-01) with medium replacement every other day until 80\% confluency, in the dark. The differentiation protocol was adapted from Zheng et al. (2019). HiPSC colonies were rapidly washed with PBS (Pan Biotech P0436500) and then incubated with accutase (Sigma, \#A6964-100ML) for 4 minutes at $37^{\circ} \mathrm{C}$. Cells were collected, centrifuged for 3 minutes at $300 \mathrm{~g}$ and resuspended in E8 flex medium containing $10 \mu \mathrm{M}$ Y27632 ROCK inhibitor (VWR, \#688000-5). Cells were counted and plated at a density of 500 cells per well in a 96-well ultra-low attachment U-bottom plate (Corning, \#CLS7007). On the following day, the medium was replaced with fresh N2-B27 medium (50\% Neurobasal (Gibco \#A3582901), 50\% DMEM/F12 (Gibco, \#11320074), 1x N2 (Gibco \#17504044), 1x B27 (Gibco, \#17504044), 1x MEM non-essential amino acids (Sigma; M7145-100ML), 1x Glutamax (Gibco, \#35050038), $0.1 \mu \mathrm{M} \beta$-mercaptoethanol (Merck Millipore \#8057400005) supplemented with 2\% Geltrex (Gibco, \#A1413301), $10 \mu \mathrm{M}$ 
1 TGF- $\beta$ pathway inhibitor (SB431542, Stem cell technology, \#72234 ) and ) $0.1 \mu \mathrm{M}$ BMP inhibitor (LDN193189, Stem cell technology, \#72147). Medium was exchanged daily. From day 4 on, organoids were cultured in $35 \mathrm{~mm}$ dishes, medium was additionally supplemented with $1 \mu \mathrm{M}$ retinoic acid (RA, Sigma, \#R2625) until day 8. At day 9, RA was removed, organoids were further cultured in N2B27 medium supplemented with LDN and SB until day 16. For organoids optogenetic stimulations, the same protocol as before was used, but the medium was supplemented with $1 \mu \mathrm{g} / \mathrm{ml}$ doxycycline to activate PA-Cre expression at day 3. On the next day, four organoids at once were embedded in a drop of Geltrex on a glass bottom dish (WillCo-dish, \#GWSB3522), incubated for $15 \mathrm{~min}$ at $37^{\circ} \mathrm{C}$ and covered with warm N2B27 medium (supplemented with SB, LDN and RA). To induce SHH expression in a restricted pole of the organoids, the laser scanning setup was chosen (Leica Sp8 SMD, see suppl. video 2): a small square $\mathrm{ROI}$ of ca. 100-400 $\mu \mathrm{M}$ was selected depending on how the four organoids were positioned with respect to each other, induced for two times 5 seconds at $100 \mathrm{~Hz}$ with $1 \%$ laser power set at $480 \mathrm{~nm}$, every 30 seconds overnight (16 hours). After induction, organoids were retrieved with a pipette and cultured individually until day 16 in an ultra-low attachment 24-well plate (Corning \#CLS3473). Control organoids were not induced. Media was exchanged daily with fresh N2B27 supplemented with SB, LDN, 2\% Geltrex and RA until day 8. From day 9 onwards, RA was removed as described before.

LED board construction and stimulation experiments.

For experimental convenience, we decided to build a custom circuit board with 96 blue LEDs that align with the used 96 well-plates. To control illumination patterns for each well individually we opted to wire each LED to a dedicated output line of a constant current LED driver chip (MAX6969). Optimizing for brightness at low supply currents to minimize excess heat, we decided on the Cree XLamp MLESBL with a documented center wavelength at $485 \mathrm{~nm}$ and a reported luminous flux of $13.9 \mathrm{~lm}$ at $50 \mathrm{~mA}$. We soldered the 96 blue LEDs onto a custom aluminum PCB. The LED PCB serves as a heat sink and is exposed to the incubator environment. A dark PVC hole mask reduces light spill. The assembly is encased in an acrylic frame and the seams sealed with neutrally curing silicone. Two cables leave the case: one to control the shift registers of the driver chips with a micro-controller outside of the incubator, and another to power the LEDs (7-9 V) and the logic chips (5V). We used the serial interface of a micro-controller (Atmel AVR ATmega32) to periodically update the shift registers of the LED drivers according to the desired patterns and to control the output 
1 latches. We opted for a control frequency of $1 \mathrm{~Hz}$ and specified the illumination patterns with a simple domain-specific language supporting four instructions: turn on the LED for up to 127 seconds (0x00...0x7E), turn off the LED for up to 127 seconds (0x80...0xFE), repeat the pattern (0x7F), and halt (0xFF). The code and the schematics for the LED board and the LED drivers are available at https://github.com/BIMSBbioinfo/casled. Stimulations were performed with a 5-seconds on, 20-seconds off pattern repeated over the desired time interval (usually 24 hours), with the cell culture plate placed directly on top of the LED board. To avoid heating, input voltage was set at $7.6 \mathrm{~V}$ for most experiments (below the optimal value for the LEDs used) and temperature of the medium in a lit 96-well plate was checked in a preliminary test with a thermocouple.

Experimental setup for parallel optogenetic stimulation (DMD setup).

Illumination from a DMD-based projector (DLP LightCrafter 4500, Texas Instruments, modified for on-axis projection by EKB technologies) was coupled to the rear port of an Observer.Z1 microscope (Zeiss), through a unity magnification relay (2x AC254-125-A-ML, Thorlabs) with an OD 2.0 neutral density filter (NE20A-A, Thorlabs). For optical stimulation, illumination from the blue $(470 \mathrm{~nm})$ LED of the LightCrafter passed through a GFP filter set (ET-GFP, Chroma, Bellow Falls, VT, USA) and projected to the sample with a 10x Plan Apo objective. For imaging of RFP, the green (530nm) LED was used together with a CY3 filter set (ET-CY3/TRITC, Chroma). Projector / camera pixel mapping and subsequent control of illumination patterns was performed using the projector plugin for Micromanager 2.0 gamma (EdelStein et al., 2014). Illumination intensity was controlled using DLP LightCrafter 4500 Control Software (v3.1.0, Texas Instruments). Emission was detected by a back illuminated sCMOS (PrimeBSI, Teledyne Photometrics). For optogenetic stimulation, samples were illuminated with $470 \mathrm{~nm}$ excitation at a power density of $4.7 \mu \mathrm{W} / \mathrm{cm}^{2}$ in user defined regions of interested (ROIs) for 20 seconds. After stimulation, full field of view RFP images were acquired. This was repeated every minute for 16 hours using a custom written Beanshell script in Micromanager. Environmental control during long term time-lapse imaging was achieved with the Incubator XLmulti S chamber and temperature/CO2 controllers (PeCon, Germany).

Laser scanning setup for single-cell stimulations.

Scanning-based optogenetic stimulation experiments were conducted using a confocal microscope Leica TCS SP8 (Leica Microsystems) equipped with an environmental $\left(\mathrm{CO}_{2}\right.$ and 
temperature) control system. Imaging and stimulation were performed using a 10x Plan APO objective and a white light laser tuned to $488 \mathrm{~nm}$ at $1 \%$ laser power. Scanning-based stimulation of $100 \times 100 \mu \mathrm{m}$ ROI containing a single cell, was performed at $100 \mathrm{~Hz}$ unidirectional scan speed. Two sequential scans were performed resulting in 10 seconds of total exposure. The stimulation protocol was repeated every 30 seconds for 16 to 20 hours. The scanning-based stimulation setup mimicked the previous LED stimulation pattern, although scanning time was set to 10 seconds, instead of 5 seconds LED illumination, in order to correct for the off-sample scan time.

RNA extraction, qRT-PCRs.

For the experiments shown in figures 2 and 4, RNA extraction was performed as follows. Cells were harvested by removing medium from 96-well plates, adding $100 \mu$ home-made Trizol directly onto the cells while keeping the plate on ice, then pipetting up and down a few times and transferring the lysate into a new $1.5 \mathrm{ml}$ tube. Lysates from two or three wells were pooled in each replicate, then RNA was extracted with the Zymo Directzol RNA miniprep kit (Zymo, \#R2051), including DNase I digestion. cDNA was synthesized using 100-200 ng RNA with the Maxima H minus RT (Thermo Fisher, \#EP0751) according to the manufacturer protocol and using random hexamers for priming. $5 \mathrm{ng}$ of diluted cDNA were used per qPCR reaction using ROX-supplemented Biozym SYBR green mastermix (Biozym, \#331416S) and $0.5 \mu \mathrm{M}$ forward and reverse primers. qPCR reactions were performed in a $A B 7500$ machine with the following cycling conditions: $95^{\circ} \mathrm{C}$ for 10 minutes, then 40 cycles of $95^{\circ} \mathrm{C}$ for 15 seconds and $60^{\circ} \mathrm{C}$ for 1 minutes with fluorescence reading, and final melting curve step.

\section{LC-MS proteomics.}

Cells were transfected and treated as described in the dedicated section. They were then checked by fluorescence after 36h for RFP knock-down and processed for proteomic analysis as follows. Cells were resuspended in $350 \mu \mathrm{l}$ of Urea buffer (8 M Urea, $100 \mathrm{mM}$ Tris-HCl, pH 8.2). Cells were lysed on a Bioruptor sonicator (Diagenode), using 10 cycles of sonication (45 sec ON, $15 \mathrm{sec}$ OFF). Protein concentration was determined by bicinchoninic acid colorimetric assay (Pierce) and a $100 \mu \mathrm{g}$ aliquot of each protein sample was reduced with $10 \mathrm{mM}$ DTT for 45 minutes at $30{ }^{\circ} \mathrm{C}$ and alkylated with $100 \mathrm{mM}$ iodoacetamide for 25 minutes at $25^{\circ} \mathrm{C}$. Proteins were digested using Lys-C (Wako, 1:40, w/w, overnight under gentle shaking at $30^{\circ} \mathrm{C}$ ) and modified trypsin (Promega, 1:60, w/w, 4 hours under rotation 
1 at $30^{\circ} \mathrm{C}$ ). Lys-C digestions product were diluted four times with $50 \mathrm{mM}$ ammonium bicarbonate before the tryptic digestion, which was stopped through acidification with $5 \mu$ of trifluoroacetic acid (Merck). Fifteen $\mu \mathrm{g}$ of each resulting peptide mixture were then desalted on Stage Tip (Rappsilber et.al., 2007), the eluates dried and reconstituted to $15 \mu \mathrm{L}$ in $0.5 \%$ acetic acid._For all the samples, 5 microliters were injected on a LC-MS/MS system (EASYnLC 1200 coupled to $Q$ Exactive HF, Thermo), using a 240 minutes gradient ranging from $2 \%$ to $50 \%$ of solvent $B(80 \%$ acetonitrile, $0.1 \%$ formic acid; solvent $A=0.1 \%$ formic acid in water). Each sample was analyzed in duplicate. For the chromatographic separation $30 \mathrm{~cm}$ long capillary (75 $\mu \mathrm{m}$ inner diameter) was packed with $1.9 \mu \mathrm{m}$ C18 beads (Reprosil-AQ, Dr. Maisch HPLC). On one end of the capillary nanospray tip was generated using a laser puller, allowing fretless packing (P-2000 Laser Based Micropipette Puller, Sutter Instruments). The nanospray source was operated with a spray voltage of $2.0 \mathrm{kV}$ and an ion transfer tube temperature of $260^{\circ} \mathrm{C}$. Data were acquired in data dependent mode, with one survey MS scan in the Orbitrap mass analyzer $(120,000$ resolution at $200 \mathrm{~m} / \mathrm{z})$ followed by up to 10 MS/MS scans $(30,000$ resolution at $200 \mathrm{~m} / \mathrm{z})$ on the most intense ions. Normalized collision energy was set to 26 , Once selected for fragmentation, ions were excluded from further selection for $30 \mathrm{~s}$, in order to increase new sequencing events. Proteomics data processing and analysis Raw data were analyzed using the MaxQuant proteomics pipeline (v1.6.10.43) and the built in the Andromeda search engine (Cox et al., 2011) with the Uniprot Human database. Carbamidomethylation of cysteines was chosen as fixed modification, oxidation of methionine and acetylation of $\mathrm{N}$-terminus were chosen as variable modifications. The search engine peptide assignments were filtered at 1\% FDR and the feature match between runs was enabled. For protein quantification LFQ intensities calculated by MaxQuant were used (Cox et al., 2014). The minimum LFQ ratio count was set to 2 and a MS/MS spectrum was always required for LFQ comparison of the precursor ion intensities. Data quality was inspected using the in-house developed tool PTXQC (Bielow et al, 2016). After removing reverse and contaminants hits, LFQ intensities were log2 transformed and proteins with less than four valid values in each condition were filtered out. Proteins with differential expression between conditions were test with Student's ttest with Benjamini-Hochberg FDR set at 0.05. Processed data are available in the supplementary table 1. 
1 Cells were treated exactly as for the proteomics experiment and as described in the dedicated section, in two additional replicates per condition. RNA was extracted with homemade Trizol by organic phase separation and RNA precipitation. Total RNA-seq libraries were performed as follows: $1 \mu \mathrm{g}$ of total RNA per sample was first depleted of ribosomal RNA using the RiboCop rRNA Depletion Kit (Lexogen, \#144) according to the manufacturer's instruction. The rRNA-depleted samples were then processed with the TruSeq mRNA stranded kit from Illumina. Libraries were then sequenced on a Nextseq with $1 \times 76$ cycles. Fastq data were generated with the bcl2fastq program and fed to the PiGx analysis pipeline (Wurmus et al., 2018), which was used with default settings with a custom reference GRCh38 human genome supplemented with two extra chromosomes carrying the CasRx-T2A-GFP cassette and the TagRFP cassette, and a custom annotation made of the Gencode v34 human annotation supplemented with two extra entries for the CasRx-T2AGFP and the TagRFP genes. For further analyses, we used the STAR/Deseq2 PiGx output. Live cell imaging for GFP and RFP quantification.

After $6,12,25$ or 50 hours of light induction or the respective dark controls, images for GFP and RFP were acquired on an inverted Nikon Ti-E microscope with a 4x NA1.4 objective and Andor iXON Ultra DU-888 camera; Z stacks had $1.5 \mu \mathrm{m}$ spacing over a $40 \mu \mathrm{m}$ range. GFP: $300 \mathrm{~ms}$ exposure; Sola $50 \%$ on $6-12 \mathrm{~h}, 12 \%$ on $25-50 \mathrm{~h}$. RFP: $100 \mathrm{~ms}$ exposure; Sola $20 \%$. All these images were taken with live cells in black 96 -well plates. Z-stacks were used for max intensity projection within imageJ, and the projection were used for signal quantification with a macro running the imageJ Subtract Background plugin with a rolling ball radius of 50 , and then the Measure function for signal intensity. This quantification assumes that all wells contain on average the same number of cells, which were seeded in the beginning of the experiment. For some of the wells, we noticed a pipetting artifact on a side, producing an area devoid of cells. We manually selected a ROI which excluded this area for all wells, and we applied this ROI before running the signal measurement macro. This experiment was performed blindly: IL transfected the cells and performed the light stimulation, then CCJ performed the imaging without knowing the samples labels, then IL ran the macros and reassigned the original labels. Immunofluorescence of hiPSCs and organoids. in a multi-well plate with agitation. For whole-mount imaging, permeabilization and blocking 
were performed for $1 \mathrm{~h}$ at room temperature in PBS solution containing $0.1 \%$ Triton-X, $0.2 \%$ BSA and 4\% normal donkey serum. Organoids were subsequently incubated with primary antibodies overnight at $4^{\circ} \mathrm{C}$ in blocking solution (PBS supplemented with $0.2 \%$ BSA and $4 \%$ normal donkey serum). The following primary antibodies were used in immunostaining: AntiFOXA2 (R\&D systems, \#AF2400; 1:100), Anti-OLIG2 (Sigma, \#HPA003254-100UL; 1:1000), Anti-NKX6.1 (Sigma, \#HPA036774-100UL; 1:500). On the following day, hiPSCs/organoids were washed 3 times for 10 minutes, with agitation, with washing solution (PBS supplemented with $0.1 \%$ Triton-X, 0.2\% BSA). Secondary antibodies and DAPI (Sigma, \#D9542) were then incubated at room temperature for $1 \mathrm{~h}$ in blocking solution. The following secondary antibodies were used at 1:1000 dilution in blocking solution: Alexa Fluor 647 anti-Rabbit (Thermo Fisher, \#A21244), Alexa Fluor 647 anti-Goat (Thermo Fisher, \#A21447), depending on the primary antibody. Samples were then washed again 3 times for 10 minutes, with agitation, in washing solution. For mounting, the organoids were placed in the center of a slide, washing solution was carefully removed and one drop of Prolong Gold antifade reagent (Thermo Fisher, \#P36930) was placed on top of each organoid. A coverslip was placed on top and the slides were allowed to dry at room temperature overnight in the dark. For hiPSCs, the mounting media was added directly in the cell culture plates were cells were seeded (Thermo Fisher, \#P10144).

For organoids slices: after fixation, organoids were allowed to settle in $1 \mathrm{~mL} 40 \%$ sucrose solution overnight at $4^{\circ} \mathrm{C}$. On the following day, they were embedded in $13 \% / 10 \%$ gelatin/sucrose solution and positioned inside an embedding mold (Sakura \#4566), rapidly moved to dry ice to freeze and then placed at $-80^{\circ} \mathrm{C}$ for storage. Blocks were removed from $-80^{\circ} \mathrm{C}$ and allowed to warm inside the cryostat to sectioning temperature $\left(-20^{\circ} \mathrm{C}\right)$ for 15 minutes. Sectioning was performed using a cryostat (Thermo Fisher Cryostar NX70) and set to produce $10 \mu \mathrm{m}$-thick slices. Cut sections were collected on slides (Thermo Fisher ,\#J1800AMNZ) and stored at $-80^{\circ} \mathrm{C}$ for long-term. To perform immunostaining, slides were allowed to warm to room temperature for 10 minutes, incubated for 5 minutes with $37^{\circ} \mathrm{C}$ PBS to remove embedding solution. Permeabilization and blocking, as well as incubation with primary and secondary antibodies, were done as described above for whole-mount organoids. 
Images were acquired using an Sp8 confocal microscope (Leica SP8 SMD) using a 10X dry or a 20x immersion objective. Z-stacks and final images were processed using Fiji-ImageJ, to produce maximal intensity projections and to subtract background.

Spatial transcriptomics experiments.

5 PET membranes (Millipore Millicell Hanging Cell Culture Insert, PET $3 \mu \mathrm{m}$, 24-well, \#MCSP24H48) were positioned in glass bottom black 24-well plate (Greiner Bio-one, \#662892), after cutting away the plastic holders, hence making the membrane touch the bottom of the well with no gaps in-between (this step was performed to ensure no light scattering or diffusion). Circular black photomasks were sticked underneath the bottom of the plate. Membranes were coated with $100 \mu \mathrm{l}$ of cold Geltrex. iPSCs were splitted to single cells, as described above, and 275,000 cells resuspended in 100ul were cultured on coated membranes generating a stable monolayer. Additional warm E8 media (300 $\mu$ l) was pipetted around the plastic scaffold. Plates were incubated at $37^{\circ} \mathrm{C}$ for $2 / 3$ hours until cells attachment. Samples were prepared in duplicates with the intent to perform control quantifications for each of those prior to the final Visium experiment. Plates were kept wrapped in aluminum foil to avoid light exposure. Before starting with the first $24 \mathrm{~h}$ of light induction, media was changed to $1 / 2$ E8 flex $+1 / 2$ COM 1 and $1 \mu \mathrm{g} / \mathrm{ml}$ of doxycycline.

The plate with the cells to be induced was covered on top by a black velvet lid and positioned onto the blue LED plate. The control sample (0h) was kept in the dark during the whole time course. Media was changed to $1 / 4$ E8 flex and 3/4 COM1 between 24h and $48 \mathrm{~h}$ of induction. Finished the time course, the 4 samples were transferred to a Visium Spatial Gene expression slide (10X Genomics) as follows: the plastic structure that surrounds the membrane was carefully held with tweezers and turned upside down to get rid of the media; membranes were delicately washed twice with $100 \mu$ of PBS and, by using a scalpel, delicately isolated from the plastic device. By using tweezers, the membranes were then slowly sticked onto a Visium Spatial Gene Expression slide with cells facing on it. The more the membrane was kept flat, the more efficient the cells transfer. The Visium Spatial Gene Expression protocol was followed, according to manufacturer instructions (10X Genomics.).

Fastq files were first processed for retrieving transcript counts with positional information with the spaceranger software (10X Genomics, v. 1.2.0). The output of spaceranger was loaded into Seurat (v. 4.0) within RStudio with R 4.0.4, and each sample was subsetted into 7 concentric circles with the center being set according to the stimulation pattern observed 
1 by fluorescent microscopy (and after checking that different radii would yield stable results in the samples with $\mathrm{SHH}$ induction, subsetting from 5 to 10 concentric circles and finally settling for an intermediate size). The central spots selected for each sample from 0 to $120 \mathrm{~h}$ had the following barcodes: CACATGATTCAGCAAC, CAATTTCGTATAAGGG, CAATTTCGTATAAGGG, GGAGGGCTTGGTTGGC (the latter north-west from the physical center as the induction was not centered). At this point, concentric circles were drawn by taking all spots with a distance from the center $<500,775,1050,1325,1600,1825$ and $>$ 1825 for the c1-c7 areas. Within these subsets of spots, the transcript counts for a $S H H$ gene set comprising SHH, NKX6-1, NKX6-2, NKX2-2, NKX2-1, FOXA2, FOXG1 and OLIG2 were added and normalized for the total transcript counts of each subset, and then further normalized by the mean of the counts for all spatial subsets c1-c7. As controls, we either randomized the genes in the gene set 1000 times, or the center spot 1000 times, and then computed an exact p-value for each subset gene set enrichment testing the hypothesis of the enrichment being larger than the random control. The signal was stable with varying binning sizes (from 6 to 9), and over cumulative analysis (Fig. S5m). Plasmids.

Supplementary table 2 contains the name, description and information on availability on the plasmids used in this study.

qRT-PCR primer pairs.

For qRT-PCR measurements of target RNAs, we used the following forward and reverse primers.

ASCL1 fw: CTTCACCAACTGGTTCTGAGG

ASCL1 rv: CAACGCCACTGACAAGAAAGC

CDR1as fw: ACGTCTCCAGTGTGCTGA

CDR1as rv: CTTGACACAGGTGCCATC

STAT3 fw: AACATGGAAGAATCCAACAACGG

STAT3 rv: TCTCAAAGGTGATCAGGTGCAG

GAPDH fw: AAGGTGAAGGTCGGAGTCAAC

GAPDH rv: GGGGTCATTGATGGCAACAATA

HPRT fw: ACCCCACGAAGTGTTGGATA 
SHH rv: ATATGTGCCTTGGACTCGTAGTAC

BMP4 fw: GCTGCTGAGGTTAAAGAGGAAACGA

BMP4 rv: CACTCGGTCTTGAGTATCCTGAG

FOXA2 fw: CCGTTCTCCATCAACAACCT

FOXA2 rv: GGGGTAGTGCATCACCTGTT

FOXG1 fw: CACTGCCTCCTAGCTTGTCC

FOXG1 rv: TGAACTCGTAGATGCCGTTG

OLIG2 fw: CCAGAGCCCGATGACCTTTTT

OLIG2 rv: CACTGCCTCCTAGCTTGTCC

NKX2-2 fw: CCGGGCCGAGAAAGGTATG

NKX2-2 rv: GTTTGCCGTCCCTGACCAA

NKX6-2 fw: GAGGACGACGACGAATACAAC

NKX6-2 rv: GTTCGAGGGTTTGTGCTTCTT

guide RNA sequences.

For most luciferase knock-downs, we used the previously validated PS18 crRNA and nontargeting control (NT) (Abuddayyeh et al., 2016) for both Psp-Cas13b and CasRx, while the complementary sequence was cloned downstream of the Renilla luciferase reporter cassette in a psiCHECK-2 plasmid (Promega). The same target sequence was also cloned downstream of a TagRFP reporter cassette in an ePB-BSD-TT piggyback vector (see plasmids) for testing constitutive and light-inducible CasRx knock-downs. For the RSD tethering experiments, the 3'UTR was further swapped with another validated protospacer sequence (Cox et al., 2017), targeting the KRAS mRNA. The CDR1as crRNAs were designed on the CDR1as backsplice junction. The STAT3 mRNA crRNA sequence was taken from Konermann et al. (2018), while the FOXA2 crRNA sequences were designed according to Wessels et al. (2020). All guide RNA sequences were cloned into the pr026 plasmid, carrying either the Psp-Cas13b or CasRx direct repeat with two adjacent Bbsl restriction sites for guide cloning (see plasmids).

NT guide: GTAATGCCTGGCTTGTCGACGCATAGTCTG PS18 guide (luciferase and TagRFP 3'UTR): CATGCCTGCAGGTCGAGTAGATTGCTGT KRAS guide (luciferase 3'UTR): AAACTATAATGGTGAATATCTTCAAATGATTT 
1 STAT3 guide: ATCACAATTGGCTCGGCCCCCATTCCCACA

2 FOXA2 PS1 guide: TAAGCCATAAATAAAGCACGCAG

3 FOXA2 PS2 guide: CAGTTTAAAATTTAACAGCCACA

4 For the light-inducible CRISPRa experiments, we used the Tet6 sgRNA spacer sequence

5 reported below, targeting CaSP1/2 and GAL4/UAS promoters. A sgRNA plasmid without

6 any spacer cloned was used as a non-targeting guide control. We report below also all the

7 tested guide RNA sequences for SHH and BMP4, designed after the Calabrese library

8 (Sanson et al., 2018). All guides were cloned into the psgRNA2.0 plasmid carrying the

9 SpCas9 sgRNA scaffold with two MS2 aptamers (Nihongaki et al., 2017, see plasmids).

10 Non-targeting guide: GAACGACTAGTTAGGCGTGTA

11 ASCL1 guide: GCAGCCGCTCGCTGCAGCAG

12 Tet6 guide: GTCTTCGGAGGACAGTACTC

13 SHH guide 1: CATCAGAAGACAAGCTTGTG

14 SHH guide 2: AAAAAACGTAGTCTTCTTCA

15 SHH guide 3: TTTCCTAAGATAAAGGTGGG

16 BMP4 guide 1: CTCGCTCGCCTCCCTTTCTG

17 BMP4 guide 2: GGGGCTCCCATCCCCAGAAA

18 BMP4 guide 3: GCCTGCTAGGCGAGGTCGGG 


\section{Bibliography (by first author alphabetical order)}

2

3

C2c2 is a single-component programmable RNA-guided RNA-targeting CRISPR effector.

Abudayyeh OO, Gootenberg JS, Konermann S, Joung J, Slaymaker IM, Cox DB, Shmakov S, Makarova KS, Semenova E, Minakhin L, Severinov K, Regev A, Lander ES, Koonin EV, Zhang F. Science. 2016 Aug 5;353(6299):aaf5573. doi: 10.1126/science.aaf5573. Epub 2016 Jun 2.

\section{RNA targeting with CRISPR-Cas13.}

Abudayyeh OO, Gootenberg JS, Essletzbichler P, Han S, Joung J, Belanto JJ, Verdine V, Cox DBT, Kellner MJ, Regev A, Lander ES, Voytas DF, Ting AY, Zhang F. Nature. 2017 Oct 12;550(7675):280-284. doi: 10.1038/nature24049. Epub 2017 Oct 4.

Proteomics Quality Control: Quality Control Software for MaxQuant Results.

Bielow C, Mastrobuoni G, Kempa S. J Proteome Res. 2016 Mar 4;15(3):777-87. doi: 10.1021/acs.jproteome.5b00780. Epub 2015 Dec 28.

RNA editing with CRISPR-Cas13.

Cox DBT, Gootenberg JS, Abudayyeh OO, Franklin B, Kellner MJ, Joung J, Zhang F. Science. 2017 Nov 24;358(6366):1019-1027. doi: 10.1126/science.aaq0180. Epub 2017 Oct 25.

Andromeda: a peptide search engine integrated into the MaxQuant environment.

Cox J, Neuhauser N, Michalski A, Scheltema RA, Olsen JV, Mann M. J Proteome Res. 2011 Apr 1;10(4):1794-805. doi: 10.1021/pr101065j. Epub 2011 Feb 22.

Accurate proteome-wide label-free quantification by delayed normalization and maximal peptide ratio extraction, termed MaxLFQ. 
1 Cox J, Hein MY, Luber CA, Paron I, Nagaraj N, Mann M.

2 Mol Cell Proteomics. 2014 Sep;13(9):2513-26. doi: 10.1074/mcp.M113.031591. Epub

32014 Jun 17.

5 Specification of positional identity in forebrain organoids.

Cederquist GY, Asciolla JJ, Tchieu J, Walsh RM, Cornacchia D, Resh MD, Studer L. Nat Biotechnol. 2019 Apr;37(4):436-444. doi: 10.1038/s41587-019-0085-3. Epub 2019 Apr 1.

Ago-TNRC6 triggers microRNA-mediated decay by promoting two deadenylation steps.

Chen CY, Zheng D, Xia Z, Shyu AB. Nat Struct Mol Biol. 2009 Nov;16(11):1160-6. doi:

10.1038/nsmb.1709. Epub 2009 Oct 18.

RNA imaging. Spatially resolved, highly multiplexed RNA profiling in single cells.

Chen KH, Boettiger AN, Moffitt JR, Wang S, Zhuang X. Science. 2015 Apr 24;348(6233):aaa6090. doi: 10.1126/science.aaa6090. Epub 2015 Apr 9.

A multifunctional AAV-CRISPR-Cas9 and its host response.

Chew WL, Tabebordbar M, Cheng JKW, Mali P, Wu EY, Ng AHM, Zhu K, Wagers AJ, Church GM. Nat Methods. 2016 Oct;13(10):868-74. doi: 10.1038/nmeth.3993. Epub 2016 Sep 5.

Self-organization of human dorsal-ventral forebrain structures by light induced $\mathrm{SHH}$.

De Santis R, Etoc F, Rosado-Olivieri AE, Brivanlou HA. bioRxiv. 2021 (preprint).

Advanced methods of microscope control using $\mu$ Manager software. 
2 Fibroblast growth factor blocks Sonic hedgehog signaling in neuronal precursors and tumor 3 cells.

4 Fogarty MP, Emmenegger BA, Grasfeder LL, Oliver TG, Wechsler-Reya RJ.

5 Proc Natl Acad Sci U S A. 2007 Feb 20;104(8):2973-8. doi: 10.1073/pnas.0605770104. Epub 2007 Feb 13.

Engineered pairs of distinct photoswitches for optogenetic control of cellular proteins.

Kawano F, Suzuki H, Furuya A, Sato M. Nat Commun. 2015 Feb 24;6:6256. doi: 10.1038/ncomms7256.

Rapid blue-light-mediated induction of protein interactions in living cells.

Kennedy MJ, Hughes RM, Peteya LA, Schwartz JW, Ehlers MD, Tucker CL. Nat Methods. 2010 Dec;7(12):973-5. doi: 10.1038/nmeth.1524. Epub 2010 Oct 31.

Multiple Input Sensing and Signal Integration Using a Split Cas12a System.

Kempton HR, Goudy LE, Love KS , Qi LS. Mol Cell. 2020 Apr 2;78(1):184-191.e3. doi: 10.1016/j.molcel.2020.01.016. Epub 2020 Feb 5.

Transcriptome Engineering with RNA-Targeting Type VI-D CRISPR Effectors. 19;173(3):665-676.e14. doi: 10.1016/j.cell.2018.02.033. Epub 2018 Mar 15.

Neural Progenitors Adopt Specific Identities by Directly Repressing All Alternative Progenitor Transcriptional Programs.

Kutejova E, Sasai N, Shah A, Gouti M, Briscoe J. Dev Cell. 2016 Mar 21;36(6):639-53. doi: 10.1016/j.devcel.2016.02.013. Epub 2016 Mar 10. 
1 Highly multiplexed subcellular RNA sequencing in situ.

2 Lee JH, Daugharthy ER, Scheiman J, Kalhor R, Yang JL, Ferrante TC, Terry R, Jeanty SS,

3 Li C, Amamoto R, Peters DT, Turczyk BM, Marblestone AH, Inverso SA, Bernard A, Mali P,

4 Rios X, Aach J, Church GM. Science. 2014 Mar 21;343(6177):1360-3. doi:

5 10.1126/science.1250212. Epub 2014 Feb 27.

Morphogen gradient reconstitution reveals Hedgehog pathway design principles.

Li P, Markson JS, Wang S, Chen S, Vachharajani V, Elowitz MB. Science. 2018 May 4;360(6388):543-548. doi: 10.1126/science.aao0645. Epub 2018 Apr 5.

Improved Tet-responsive promoters with minimized background expression.

Loew R, Heinz N, Hampf M, Bujard H, Gossen M. BMC Biotechnol. 2010 Nov 24;10:81. doi: $10.1186 / 1472-6750-10-81$.

Spatiotemporal dynamics of molecular pathology in amyotrophic lateral sclerosis.

Maniatis S, Äijö T, Vickovic S, Braine C, Kang K, Mollbrink A, Fagegaltier D, Andrusivová Ž, Saarenpää S, Saiz-Castro G, Cuevas M, Watters A, Lundeberg J, Bonneau R, Phatnani H. Science. 2019 Apr 5;364(6435):89-93. doi: 10.1126/science.aav9776.

Light-dependent RNA interference with nucleobase-caged siRNAs.

Mikat V, Heckel A. RNA. 2007 Dec;13(12):2341-7. doi: 10.1261/rna.753407. Epub 2007 Oct 19.

Museum of Spatial Transcriptomics.

Moses L, Pachter L. bioRxiv, 2021.

A novel phosphorylation-independent interaction between SMG6 and UPF1 is essential for human NMD. 
1 Nicholson P, Josi C, Kurosawa H, Yamashita A, Mühlemann O. Nucleic Acids Res. 2014 Aug;42(14):9217-35. doi: 10.1093/nar/gku645. Epub 2014 Jul 22.

Photoactivatable CRISPR-Cas9 for optogenetic genome editing.

Nihongaki Y, Kawano F, Nakajima T, Sato M. Nat Biotechnol. 2015 Jul;33(7):755-60. doi:

10.1038/nbt.3245. Epub 2015 Jun 15.

CRISPR-Cas9-based photoactivatable transcription system.

Nihongaki Y, Yamamoto S, Kawano F, Suzuki H, Sato M. Chem Biol. 2015 Feb 19;22(2):169-74. doi: 10.1016/j.chembiol.2014.12.011. Epub 2015 Jan 22.

CRISPR-Cas9-based photoactivatable transcription systems to induce neuronal differentiation.

Nihongaki Y, Furuhata Y, Otabe T, Hasegawa S, Yoshimoto K, Sato M. Nat Methods. 2017 Oct;14(10):963-966. doi: 10.1038/nmeth.4430. Epub 2017 Sep 11.

Gene expression cartography.

Nitzan M, Karaiskos N, Friedman N, Rajewsky N. Nature. 2019 Dec;576(7785):132-137. doi: 10.1038/s41586-019-1773-3. Epub 2019 Nov 20.

Optoribogenetic control of regulatory RNA molecules.

Pilsl S, Morgan C, Choukeife M, Möglich A, Mayer G. Nat Commun. 2020 Sep 24;11(1):4825. doi: 10.1038/s41467-020-18673-5.

A light-inducible CRISPR-Cas9 system for control of endogenous gene activation.

Polstein LR, Gersbach CA. Nat Chem Biol. 2015 Mar;11(3):198-200. doi: 10.1038/nchembio.1753. Epub 2015 Feb 9. 
1 Protocol for micro-purification, enrichment, pre-fractionation and storage of peptides for

3 Rappsilber J, Mann M, Ishihama Y. Nat Protoc. 2007;2(8):1896-906. doi:

$4 \quad 10.1038 /$ nprot.2007.261.

5

A LOV2 domain-based optogenetic tool to control protein degradation and cellular function. Renicke C, Schuster D, Usherenko S, Essen LO, Taxis C. Chem Biol. 2013 Apr 18;20(4):619-26. doi: 10.1016/j.chembiol.2013.03.005.

Establishing and interpreting graded Sonic Hedgehog signaling during vertebrate neural tube patterning: the role of negative feedback.

Ribes V, Briscoe J. Cold Spring Harb Perspect Biol. 2009 Aug;1(2):a002014. doi: 10.1101/cshperspect.a002014.

Slide-seq: A scalable technology for measuring genome-wide expression at high spatial resolution.

Rodriques SG, Stickels RR, Goeva A, Martin CA, Murray E, Vanderburg CR, Welch J, Chen LM, Chen F, Macosko EZ. Science. 2019 Mar 29;363(6434):1463-1467. doi: 10.1126/science.aaw1219. Epub 2019 Mar 28.

Optimized libraries for CRISPR-Cas9 genetic screens with multiple modalities.

Sanson KR, Hanna RE, Hegde M, Donovan KF, Strand C, Sullender ME, Vaimberg EW, Goodale A, Root DE, Piccioni F, Doench JG. Nat Commun. 2018 Dec 21;9(1):5416. doi: 10.1038/s41467-018-07901-8.

In Situ Transcription Profiling of Single Cells Reveals Spatial Organization of Cells in the Mouse Hippocampus. 
1 Shah S, Lubeck E, Zhou W, Cai L. Neuron. 2016 Oct 19;92(2):342-357. doi: 10.1016/j.neuron.2016.10.001.

Visualization and analysis of gene expression in tissue sections by spatial transcriptomics.

Ståhl PL, Salmén F, Vickovic S, Lundmark A, Navarro JF, Magnusson J, Giacomello S, Asp

M, Westholm JO, Huss M, Mollbrink A, Linnarsson S, Codeluppi S, Borg Å, Pontén F,

Costea PI, Sahlén P, Mulder J, Bergmann O, Lundeberg J, Frisén J. Science. 2016 Jul 1;353(6294):78-82. doi: 10.1126/science.aaf2403.

LOVTRAP: an optogenetic system for photoinduced protein dissociation.

Wang H, Vilela M, Winkler A, Tarnawski M, Schlichting I, Yumerefendi H, Kuhlman B, Liu Epub 2016 Jul 18.

Genome-wide analysis of PDX1 target genes in human pancreatic progenitors.

Wang X, Sterr M, Burtscher I, Chen S, Hieronimus A, Machicao F, Staiger H, Häring HU, Lederer G, Meitinger T, Cernilogar FM, Schotta G, Irmler M, Beckers J, Hrabě de Angelis M, Ray M, Wright CVE, Bakhti M, Lickert H. Mol Metab. 2018 Mar;9:57-68. doi: 10.1016/j.molmet.2018.01.011. Epub 2018 Jan 31.

Massively parallel Cas13 screens reveal principles for guide RNA design.

Wessels HH, Méndez-Mancilla A, Guo X, Legut M, Daniloski Z, Sanjana NE. Nat

Biotechnol. 2020 Jun;38(6):722-727. doi: 10.1038/s41587-020-0456-9. Epub 2020 Mar 16.

PiGx: reproducible genomics analysis pipelines with GNU Guix.

Wurmus R, Uyar B, Osberg B, Franke V, Gosdschan A, Wreczycka K, Ronen J, Akalin A. Gigascience. 2018 Dec 1;7(12):giy123. doi: 10.1093/gigascience/giy123. 
1 Spatial transcriptome profiling by MERFISH reveals subcellular RNA compartmentalization and cell cycle-dependent gene expression.

Xia C, Fan J, Emanuel G, Hao J, Zhuang X. Proc Natl Acad Sci U S A. 2019 Sep 24;116(39):19490-19499. doi: 10.1073/pnas.1912459116. Epub

Light Control of the Tet Gene Expression System in Mammalian Cells.

Yamada M, Suzuki Y, Nagasaki SC, Okuno H, Imayoshi I. Cell Rep. 2018 Oct 9;25(2):487500.e6. doi: 10.1016/j.celrep.2018.09.026.

A split-Cas9 architecture for inducible genome editing and transcription modulation.

Zetsche B, Volz SE, Zhang F. Nat Biotechnol. 2015 Feb;33(2):139-42. doi:

$10.1038 / \mathrm{nbt} .3149$.

Structural Basis for the RNA-Guided Ribonuclease Activity of CRISPR-Cas13d.

Zhang C, Konermann S, Brideau NJ, Lotfy P, Wu X, Novick SJ, Strutzenberg T, Griffin PR, Hsu PD, Lyumkis D. Cell. 2018 Sep 20;175(1):212-223.e17. doi:

10.1016/j.cell.2018.09.001.

Dorsal-ventral patterned neural cyst from human pluripotent stem cells in a neurogenic niche.

Zheng Y, Xue X, Resto-Irizarry AM, Li Z, Shao Y, Zheng Y, Zhao G, Fu J. Sci Adv. 2019 Dec 11;5(12):eaax5933. doi: 10.1126/sciadv.aax5933. eCollection 2019 Dec.

A Single-Chain Photoswitchable CRISPR-Cas9 Architecture for Light-Inducible Gene Editing and Transcription.

Zhou XX, Zou X, Chung HK, Gao Y, Liu Y, Qi LS, Lin MZ. ACS Chem Biol. 2018 Feb 16;13(2):443-448. doi: 10.1021/acschembio.7b00603. Epub 2017 Sep 29. 


\section{$1 \quad$ Figures and legends}

\section{$3 \quad$ Figure 1}

a

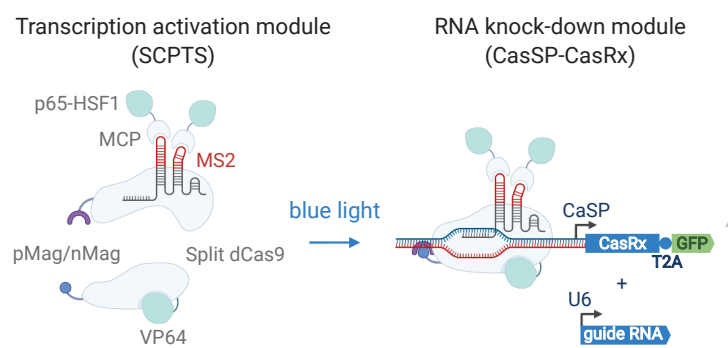

C

Transfection / transduction Photostimulation GFP imaging

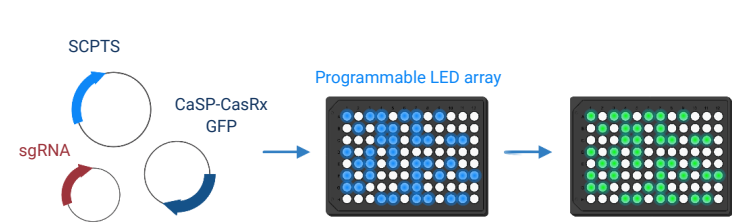

e

Figure 1. Light-inducible transcription of CasRx. b

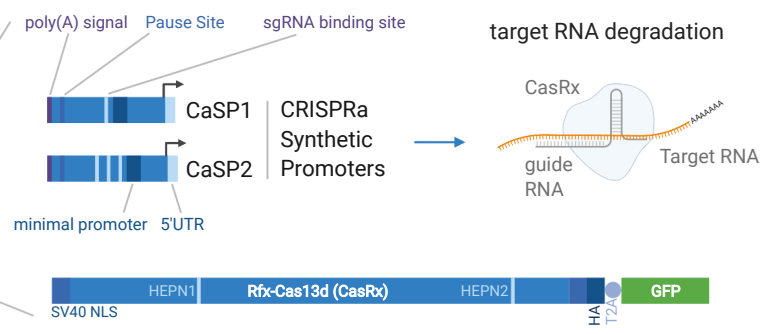

d

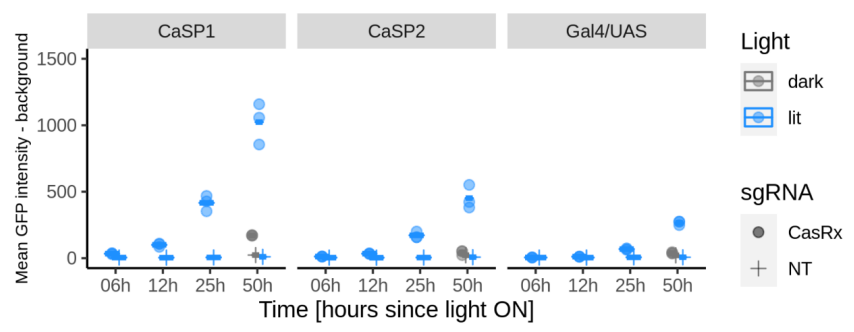

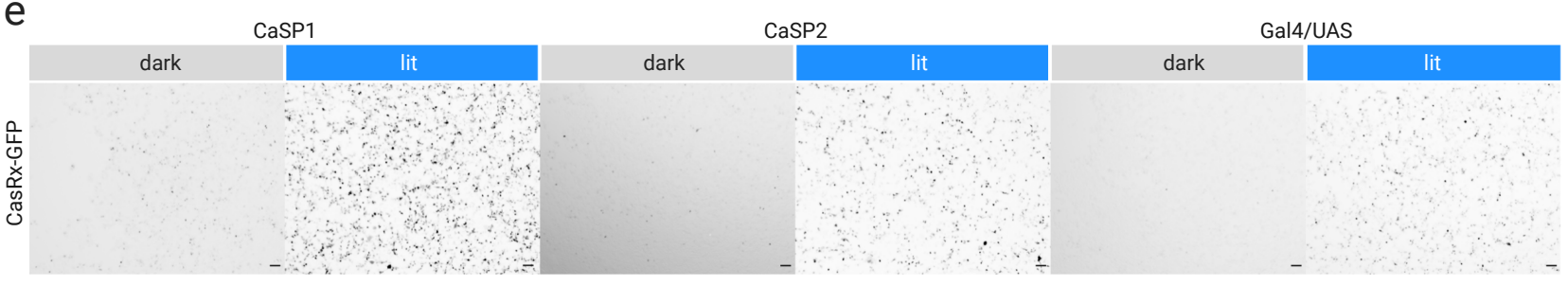

a. Left: light-inducible transcription activation module (SCPTS, Nihongaki et al., 2017). The Cas9 sgRNA is shown associated to the $\mathrm{N}$-ter part of dCas9 in the dark state. Right: RNA knock-down module: CasRx transcription is driven by a synthetic promoter controlled by the SCPTS module. A guide RNA for targeted knock-downs can be co-expressed from a U6 promoter. $\mathbf{b}$. Synthetic promoters for light-inducible transcription of CasRx (CaSP1/2), containing upstream elements for reducing spurious transcription (poly(A) site, pause site), a minimal CMV promoter containing TFIIB binding site/TATA box, an initiator and synthetic 5'UTR, one or three sgRNA binding sites. The CasRx cassette (below) contains a T2A-GFP tag, two nuclear localization signals (NLS) and an HA tag, as in Konermann et al. (2018). HEPN1-2 (Higher Eukaryotes and Prokaryotes Nucleotide-binding) catalytic domains are indicated. c. Experimental setup: HEK293T cells are transfected in a 96-well plate, which is placed on a LED-board for blue-light stimulation. At each time point, cells are imaged for GFP quantification. d. For the SCPTS system, background-subtracted mean GFP intensity is plotted for the selected time points (dark or lit), with one of the three promoters (CaSP1/2, Gal4/UAS), with either a nontargeting guide (NT) or the CasRx promoter-targeting guide (CasRx). Horizontal bars: mean of all replicates (three). g. Representative images for the SCPTS CasRx system with the three promoters in presence of the CasRx sgRNA. Scale bar: $100 \mu \mathrm{m}$. Images were taken at $24 \mathrm{~h}$ post-transfection with a Keyence BZ-X710 with $4 \mathrm{x}$ magnification, in independent experiments from those quantified in panel $\mathrm{d}$. 


\section{$1 \quad$ Figure 2}

a

Knock-downs with CasRx
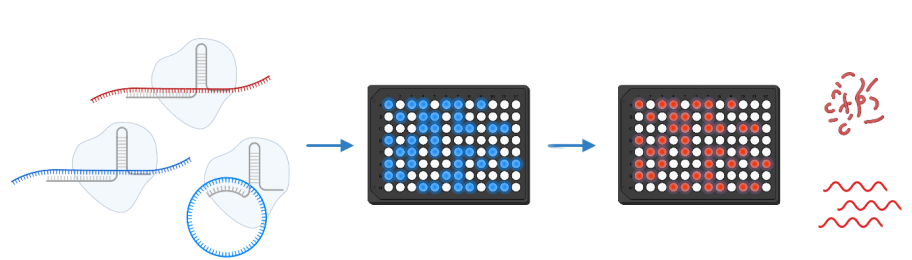

d

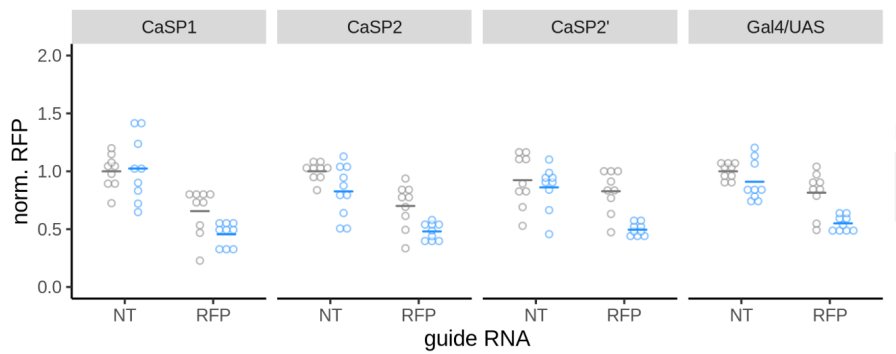

b

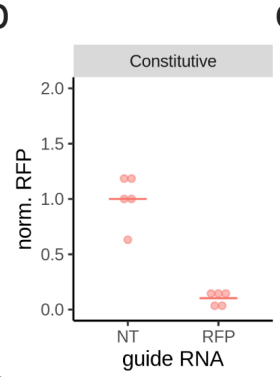

e

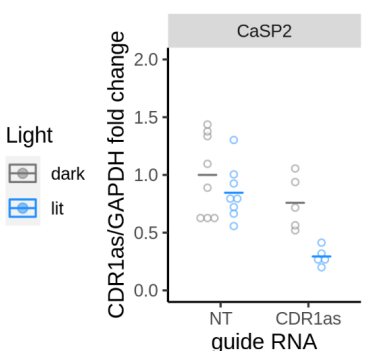

C
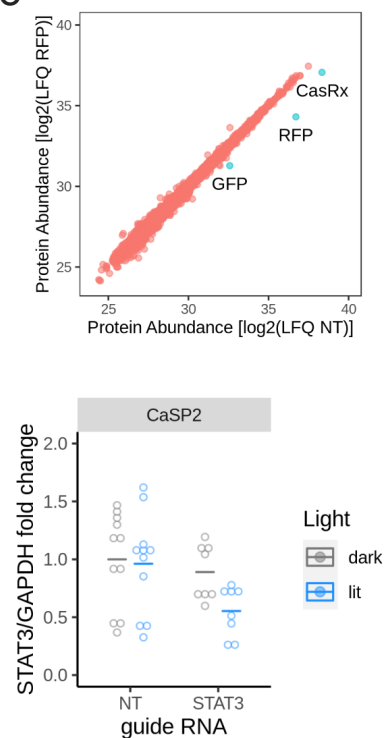

Figure 2. Light-inducible knock-down of target RNA.

a. CasRx knock-downs of reporter and endogenous target RNAs, along with readouts of efficiency, offtargeting and leakage. b. Background-subtracted RFP signal intensity normalized on the non-targeting guide control (NT), with a constitutively expressed CasRx cotransfected with the RFP reporter, a non-targeting guide or an RFP-targeting guide (RFP). Horizontal bars: mean of all replicates (three). c. Protein abundance (average over 6 replicates: three biological times two technical) in cells transfected with a constitutively expressed CasRx, the RFP reporter, a non-targeting guide (NT) or an RFP-targeting guide (RFP). Protein abundance was measured by shot-gun proteomics. LFQ: log2 Label-Free Quantification intensity. Green: proteins with statistically significant and $>$ two-fold change. $d$. As in b, for the light-inducible promoters indicated on top of each panel used in combination with the SCPTS system. Grey and blue: dark and lit, horizontal bars: mean of all replicates (9 for all, 10 for one condition). CaSP1, CaSP2 and CaSP2' (same as CaSP2 but with a CasRx devoid of the GFP tag) had a significant difference in fluorescence intensity between dark and lit conditions for the RFP-targeting guide ( $p$-value $<0.05$, Student's $t$ test). All systems had non-significant difference between dark and lit conditions with the non-targeting guide. e. qRT-PCR for CDR1as /STAT3, normalized to GAPDH and the non-targeting guide control as the reference sample for normalization, for cells treated with the CaSP2 CasRx light-inducible system along with either a non-targeting guide (NT) or one CDR1as-targeting guide (CDR1as), or a STAT3-targeting guide (STAT3). Grey, blue colors: dark, lit. horizontal bars: mean over each condition. $p$-value $<0.05$ in dark vs lit, Student's $t$ test. 


\title{
$1 \quad$ Figure 3
}

a

Transfection / transduction
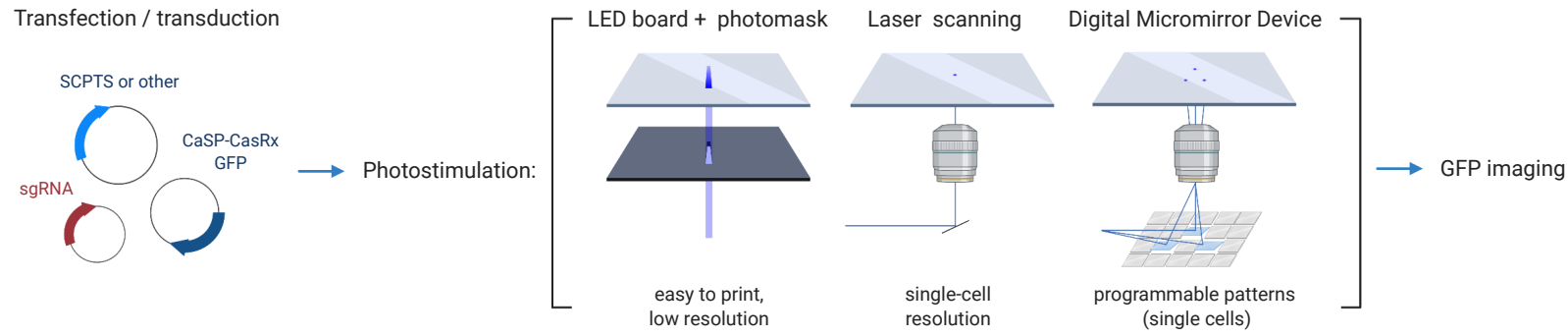

b

C

Laser scanning

d
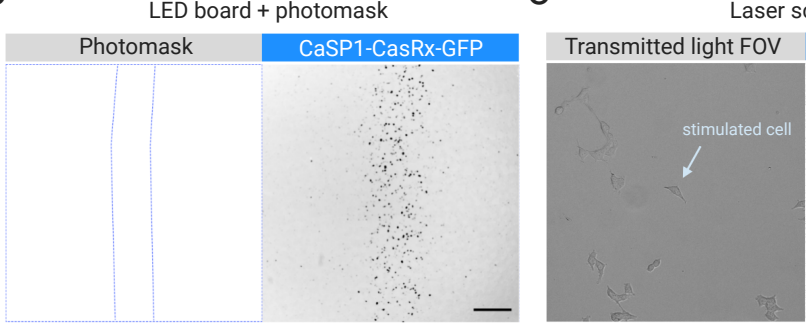

\begin{abstract}
DMD
\end{abstract} DMD ROIs $\quad$ PA-Cre/Lox-CasRx-GFP

4 Figure 3. Spatial patterning of CasRx activation.

a. Spatially induced photo-stimulations: cells are stimulated with a blue LED array combined with a black photomask, or with a confocal microscope setup by laser scanning, or with a Digital Micromirror Device microscope by a LED source projected through a micromirror array to the cell culture plate bottom. b. Representative fluorescent microscope image of a photomask stimulation of the CaSP1-CasRx system in HEK cells. Blue: photomask shape. Signal is GFP in grey scale. Scale bar: $500 \mu \mathrm{m}$. c. Representative image of a single-cell Cre/Lox CasRx stimulation in HEK cells, performed with $100 \mathrm{~Hz}$ laser scanning within a confocal microscope setup. Left: transmitted light image, right: confocal image of the GFP signal in grey scale. Scale bar: $100 \mu \mathrm{m}$. d. Representative image of a complex pattern stimulation of the Cre/Lox CasRx system in hiPSCs performed with the DMD setup. The selected ROls are shown on the left, while the NeonGreen signal imaged with a confocal setup after $24 \mathrm{~h}$ stimulation within the DMD setup is shown in grey scale on the right. Scale bar: $100 \mu \mathrm{m}$. 
a Transfection Sonic Hedgehog photostimulation
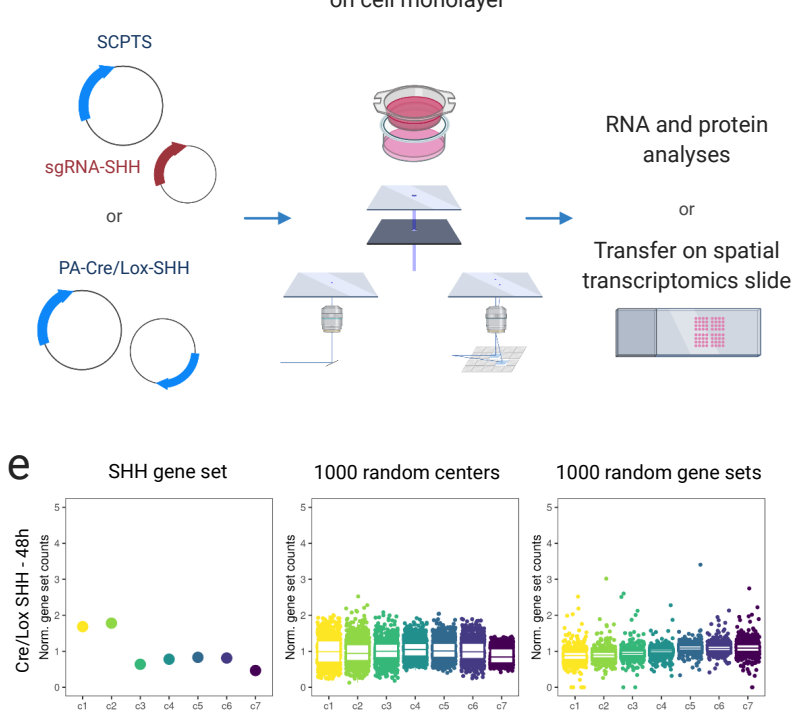

1000 random centers

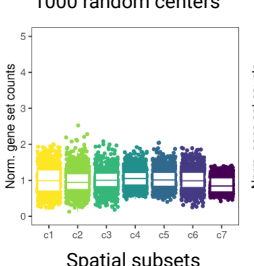

f

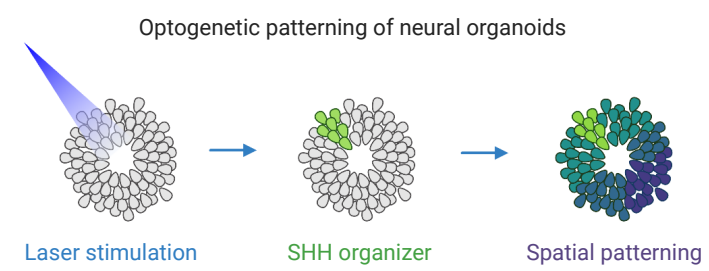

1000 random gene sets b

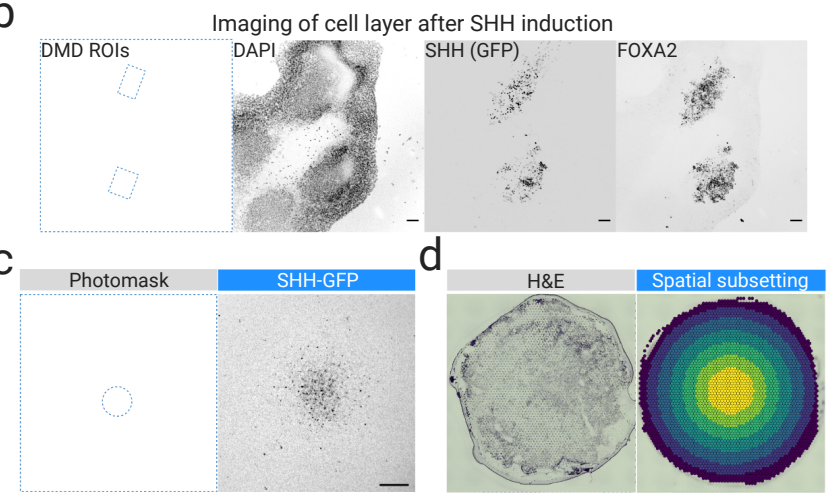
toon random gene sets

g

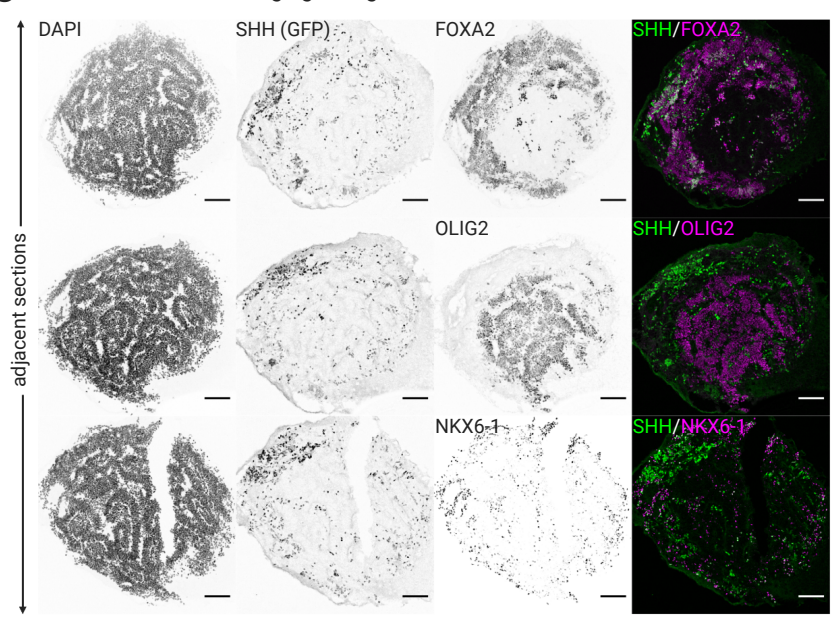

Figure 4. Optogenetic stimulation of Sonic Hedgehog in human stem cells and organoids.

a. Coupled optogenetic stimulation of SHH and spatial readouts. b. Imaging of DAPI, SHH-expressing cells marked by NeonGreen (labeled as GFP for simplicity) and FOXA2 (immunofluorescence) in hiPSCs. SHH wasin the two ROls on the left with the DMD setup and then cultured in neural induction media for 6 days. Signal is shown in greyscale for each channel. Scale bar: $100 \mu \mathrm{m}$. c. Representative image of hiPSCs Cre/Lox $S H H$ cultured as a monolayer on a PET membrane, induced in the center with a $500 \mu m$-wide circular photomask (left). Signal is shown in greyscale (right). Scale bar: $500 \mu \mathrm{m}$. d. Left: representative H\&E staining of a hiPSC layer cultured on a membrane and transferred onto a VISIUM slide. Right: representative spatial subsetting of spots within a capture area of a VISIUM slide into 7 concentric circles, centered on the $\mathrm{SHH}$ induced area. e. Left: normalized counts of a SHH gene set (SHH, FOXA2, FOXG1, NKX2-1, NKX2-2, NKX62, NKX6-1, OLIG2) in the 7 concentric circles c1-7, color coded as in d, in hiPSCs stimulated for 48h. Middle: same as left, sampling 1000 times a random central spot. Right: same as left, sampling 1000 times a random gene set. C1-2 were significant over both spatial and gene set sampling ( $p$-value $<0.05$ ). $f$. Optogenetic patterning of neural organoids: an embryoid body expressing the PA-Cre/Lox-SHH-GFP system is photostimulated in a restricted area via laser scanning. The resulting organizer, made of $\mathrm{SHH}$-expressing cells, instructs the neighboring cells to form distinct spatial domains of gene expression. g. Imaging of DAPI, SHHexpressing cells marked by NeonGreen (GFP for simplicity) and FOXA2/OLIG2/NKX6-1 in adjacent cryosections of neural organoids with laser induction of $\mathrm{SHH}$ in the north-west pole. Signal in grey scale for each target separately, and merged in green and magenta (right). Scale bar: $100 \mu \mathrm{m}$. Experiment was performed in 4 replicates and representative images are shown here. 